\title{
Efficacy of Psychological Interventions on Depression Anxiety and Somatization in Migrants: A Meta-analysis
}

\author{
Daniela Sambucini $^{1} \cdot$ Paola Aceto $^{2,3} \cdot$ Edvaldo Begotaraj $^{1} \cdot$ Carlo Lai $^{1}$ (1)
}

Published online: 25 July 2020

(c) The Author(s) 2020

\begin{abstract}
Many studies reveal the effectiveness of different psychological interventions on the adult refugees reporting mental health distress. Aim of this metanalysis was to test the efficacy of different psychological treatments on the depressive, anxiety and somatization symptoms on refugees and asylum seekers. Fifty-two studies, since 1997 to 2019, were included in the systematic review and 27 of those were included in the metanalysis. Studies providing a pre and post treatment methodological design were included. All treatments reported significant effects on the three outcomes. Qualitative observations showed a probability to have a significant pre-post treatment effects on trials with outcome of depression (56\%), anxiety (44\%), and somatization (42\%). Cognitive behavioral treatment resulted the most effective treatment. The status of refugee compared to the status of asylum seeker seems to have a great effect on the effectiveness of the treatment.
\end{abstract}

Keywords Migration $\cdot$ Psychological intervention $\cdot$ Depression $\cdot$ Anxiety $\cdot$ Somatization

\section{Introduction}

Geopolitical migration caused the emergency to individuate an efficacy intervention for treating the traumatic symptomatology of the migrants [1]. The migrants often originate from countries with governate conditions of war or great poorness that increase the psychological distress [2,3].

According UNHCR data of the 2015 the number of applications for asylum increased in Europe more than doubling from 2013 [4, 5]; in this period 17 million of refugees and asylum seekers were outside their counties of origin [6].

From 2013 to 2015 Italy State responded positively to about $40 \%$ of asylum applications, a percentage that was lowered in 2016 [7].

Electronic supplementary material The online version of this article (https://doi.org/10.1007/s10903-020-01055-w) contains supplementary material, which is available to authorized users.

Carlo Lai

carlo.lai@uniroma1.it

1 Department of Dynamic and Clinical Psychology, Sapienza University of Rome, Via degli Apuli, 1, 00185 Rome, Italy

2 A. Gemelli University Polyclinic, IRCSS Foundation, Rome, Italy

3 Sacred Heart Catholic University, Rome, Italy
The number of people in the world who suffer from political violence and war pursued by the war seems to grow by about $1 \%$ every year [8].

The nature of conflict changed in last 20 years considering that the current war victims are civilians rather than combatants [3] causing a phenomenon of forced migration that represents, in many cases, the only option to survive. The forced migration, due to politics or war reasons produces unmistakable psychological signs [5]. Migrants experienced multiple stressful events before, during and after their travel: imprisonment, rape, ethnic cleansing, physical violence and torture. They often witnessed violence against or the death of loved ones [2]; persecutions, bereavement in their origin countries and discrimination in their host countries that contribute, successively, them to develop a "re-traumatization" post migration [5].

The exposure to interpersonal violence associated with emotional, sexual and physical abuse, torture and exploitation, and other atrocities committed in war, cause psychological distress that have strong impact on survivors life. Many psychological sequelae as destructive behaviors, substances misuse, self-harm, unsafe sexual practices and involvement in abusive relationship seems to be due to these traumatic conditions [9].

Moreover, forced migrants could experience deaths or suicides of their loved people, parents, kin, sons etc. These 
bereavements could expose the survivor migrant to thoughts of death, to a probability of exacerbation of the psychopathological symptoms that could last many years, and also to suicidal attempts. For this reason, it seems very important to explore what happens after the death or the suicide of a significant other, so to provide a proper and ad hoc care [10]. According to this clinical issue, it is important the knowledge of the neurobiological factors that underlie the suicide risk. Recent findings [11] showed an association between a biological dysregulation with the suicide attempts. This dysregulation has been interpreted as a compensatory mechanism that involves the prolactin and the thyroid hormones, useful to corrects the reduced central serotonin activity. In the next future this neurobiological correlate could be useful to individuate a suicide risk in the clinical practice [11]. The displacement's perception, the events that caused the migration and the hostility in the host country post migration (culture shock), seem to decrease life expectancy, and produce insecurity, isolation and poorness, causing the development of mental illness including anxiety, depression and somatization [5].

In the last 20 years many types of psychological treatments - combined or not with pharmacological treatmenthave been applied in migrants showing to be efficient [8-12].

Nowadays there are different systematic reviews that summarize the great number of studies on the efficacy of psychological intervention to post-migration symptoms. However, the four metanalysis published in the last ten years are focused on specific post-traumatic stress disorder PTSD outcome. The large number of studies published on this field allowed to perform metanalysis on specific therapeutic approaches and on specific psychological dimensions.

Aim of the present study was to assess, in adult migrants, the outcome of the main psychological interventions on post migration specific symptomatology: depression, anxiety and somatization.

\section{Method}

\section{Search Strategy and Data Sources}

The present systematic review and metanalysis has been registered on the International Prospective Register of Systematic Reviews (PROSPERO).

The literature search was conducted on health data base that included: Pubmed, Scholar, Psych INFO, Published International Literature on Traumatic Stress (PILOT). Additional studies were identified by cross-referencing.

\section{Inclusion Criteria and Selection of Studies}

The search strategy was based on the following main search components: "migrants", "refugees", "asylum seekers" (alone and combined). All randomized controlled trials (RCT), multiple perspective cohort trials (MCT) and single perspective cohort trials (SCT) written in English and assessing psychological and/or pharmacological intervention with pre and post evaluation of depression, anxiety, somatization were considered eligible for inclusion. Only experimental groups investigating psychological interventions were considered for comparisons. The control groups were eliminated as they included waiting lists or usual treatments. Included studies had to report at least one quantitative measure of depression and/or anxiety and/or somatization assessed before and after treatment.

\section{Data Extraction and Coding}

A qualitative systematic review for all 52 studies was performed (Table 1).

In the first metanalysis a comparison between pre-treatment and post-treatment values was been performed for each outcome (depression, anxiety, somatization) differentiating the trials based on research design (RCT, MCT, SCT). When two or more measurement scales for the same outcome (for example Hamilton Depression Rating Scale and Beck Depression Inventory) were administered in the same trial, the more frequent scale among all trials included in the meta-analysis was considered. Further sub-analysis, were made dividing the trials according 3 variables: status (refugees, asylum seekers), provenance (Middle East, Africa, Asia, Latin America), and treatment (narrative exposition therapies-NET, cognitive behavioural treatment-CBT, Dynamic therapies, combined psychological treatments, combined pharmacological/psychological treatments). The metanalysis was performed when at least 4 trials were homogeneous for the considered variables.

\section{Heterogeneity}

Evaluation of heterogeneity was reported by the RevMan5.3 program. When the heterogeneity was high (despite the significance of the result), further sub-analysis (status, provenance, single treatment) were made.

\section{Mean Differences}

Pre and post intervention data of all the experimental treatments (within group) were inserted to calculate the means difference. For each trial mean difference, standard 


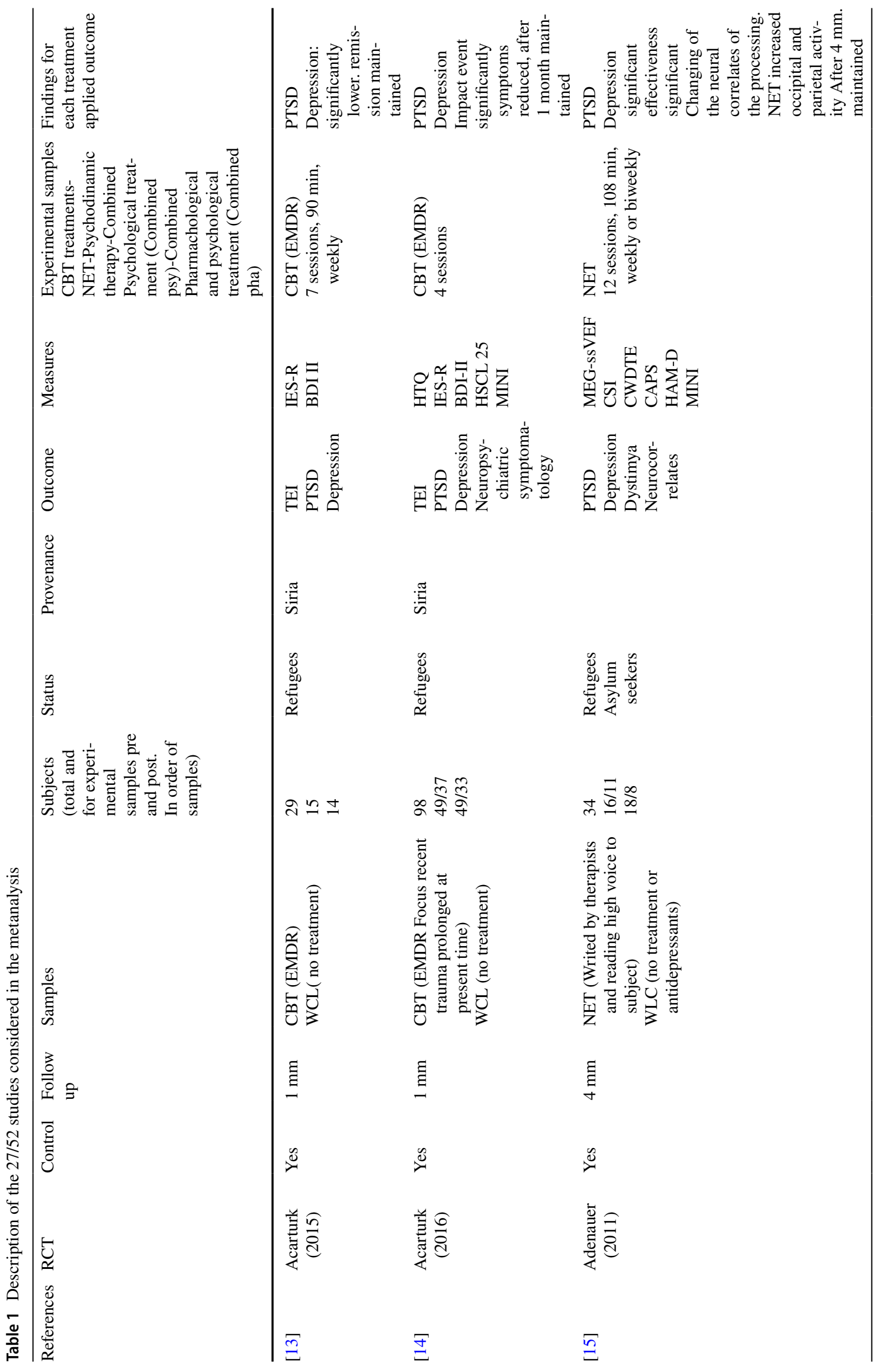




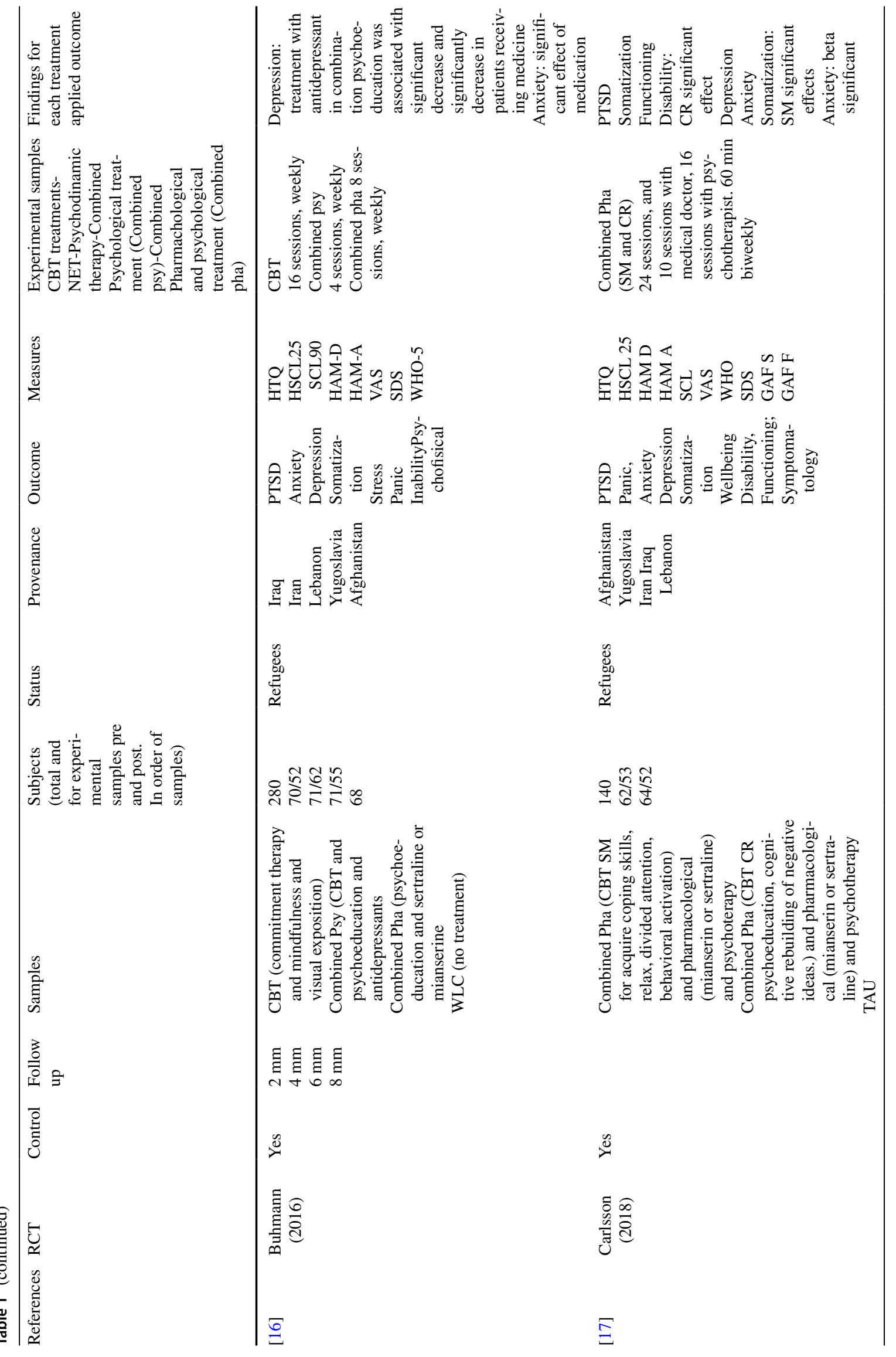




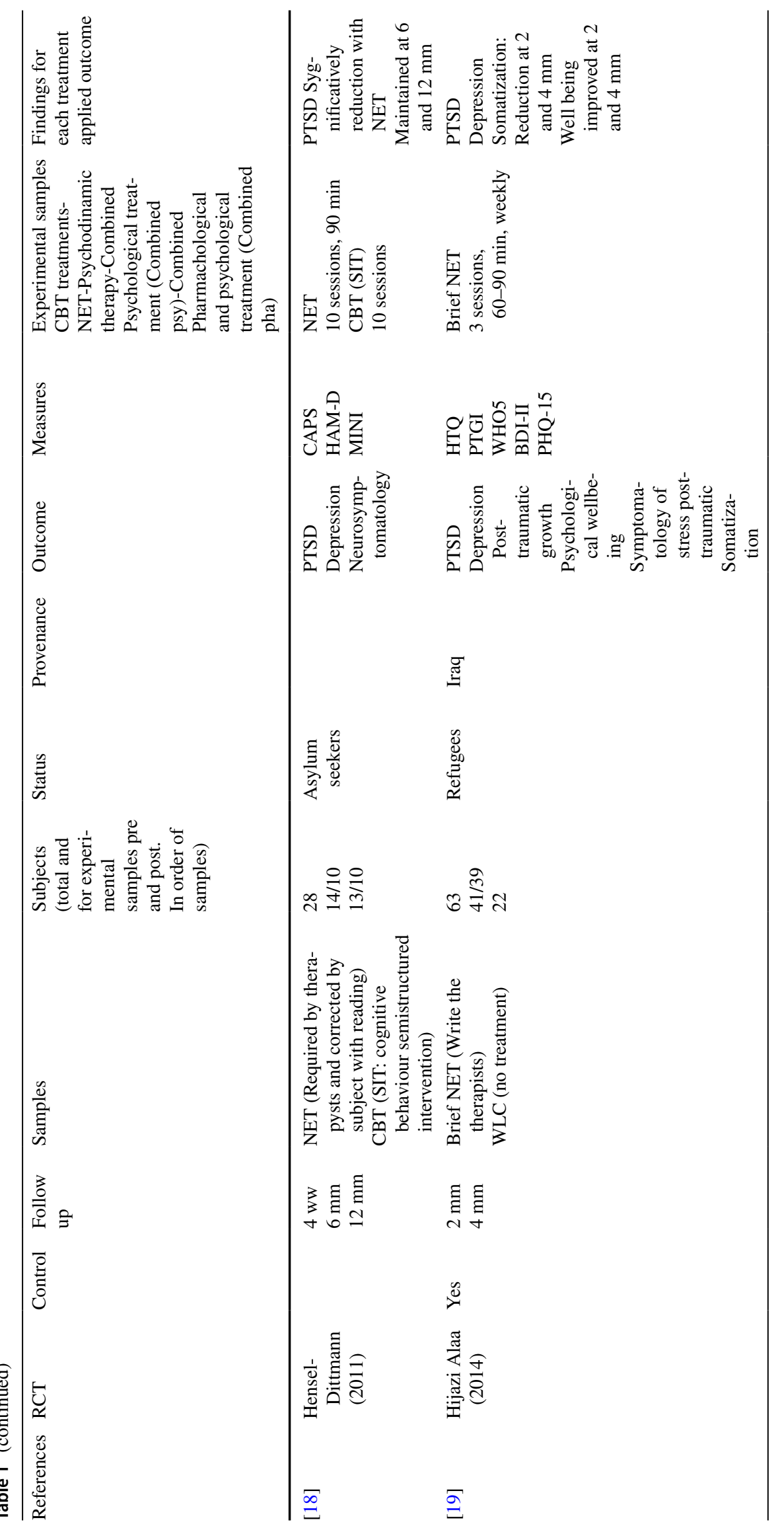




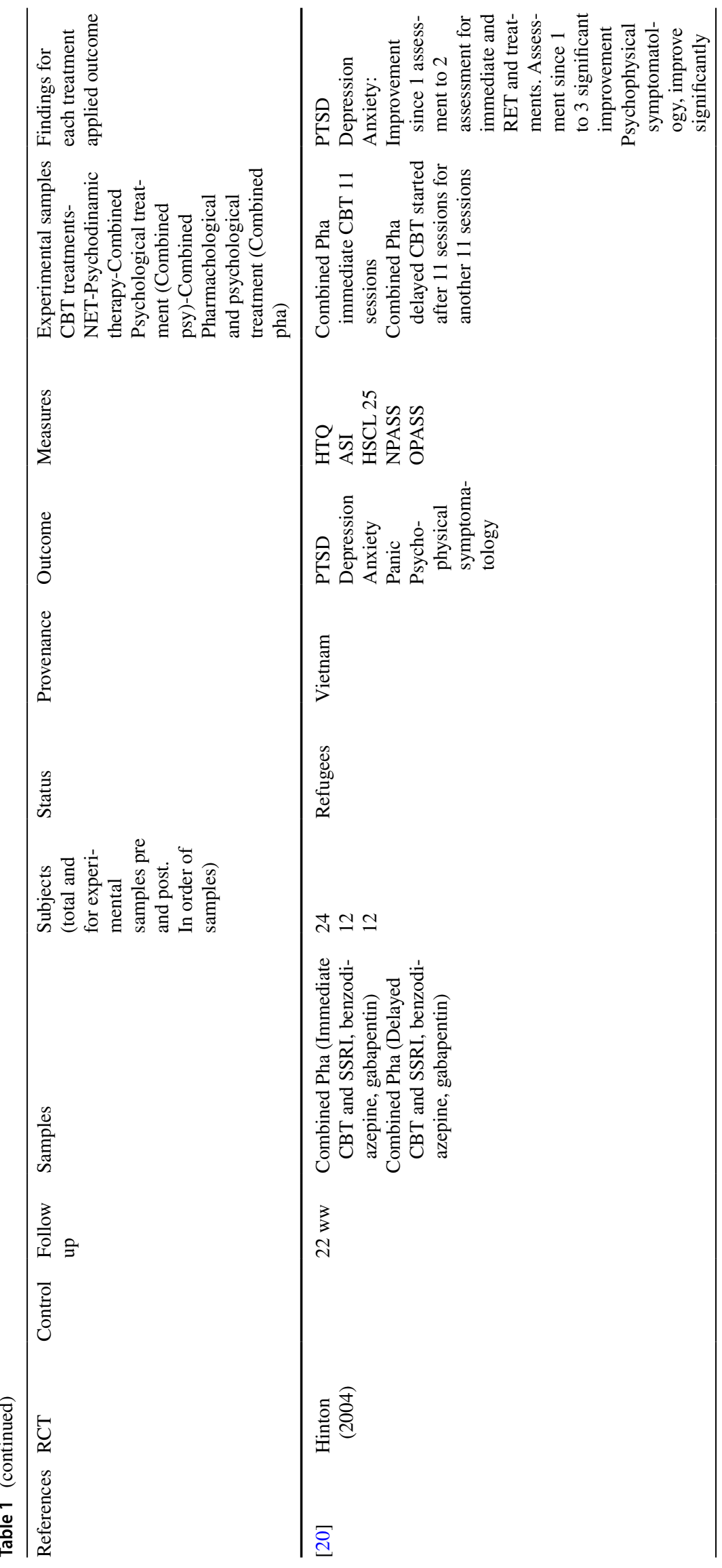




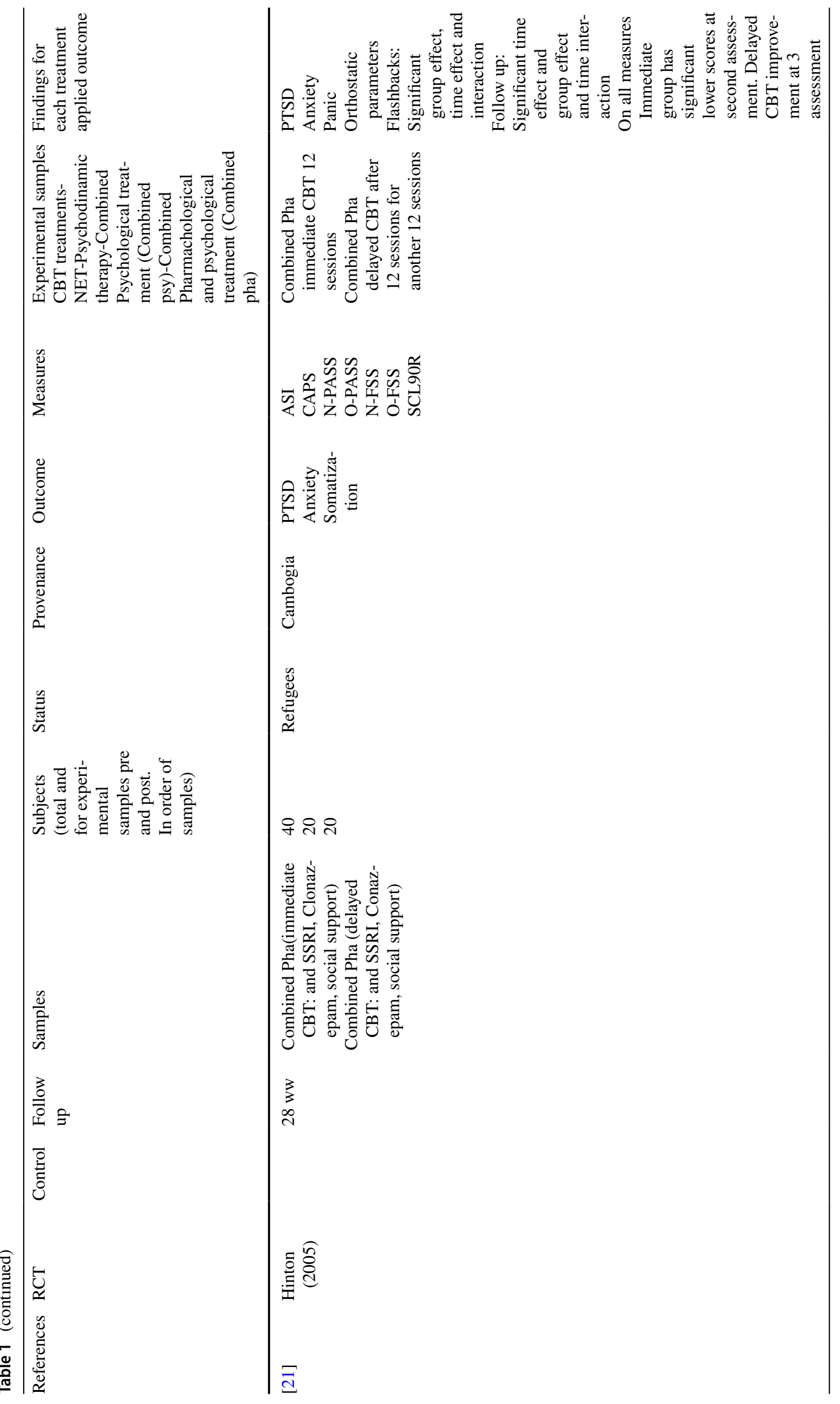




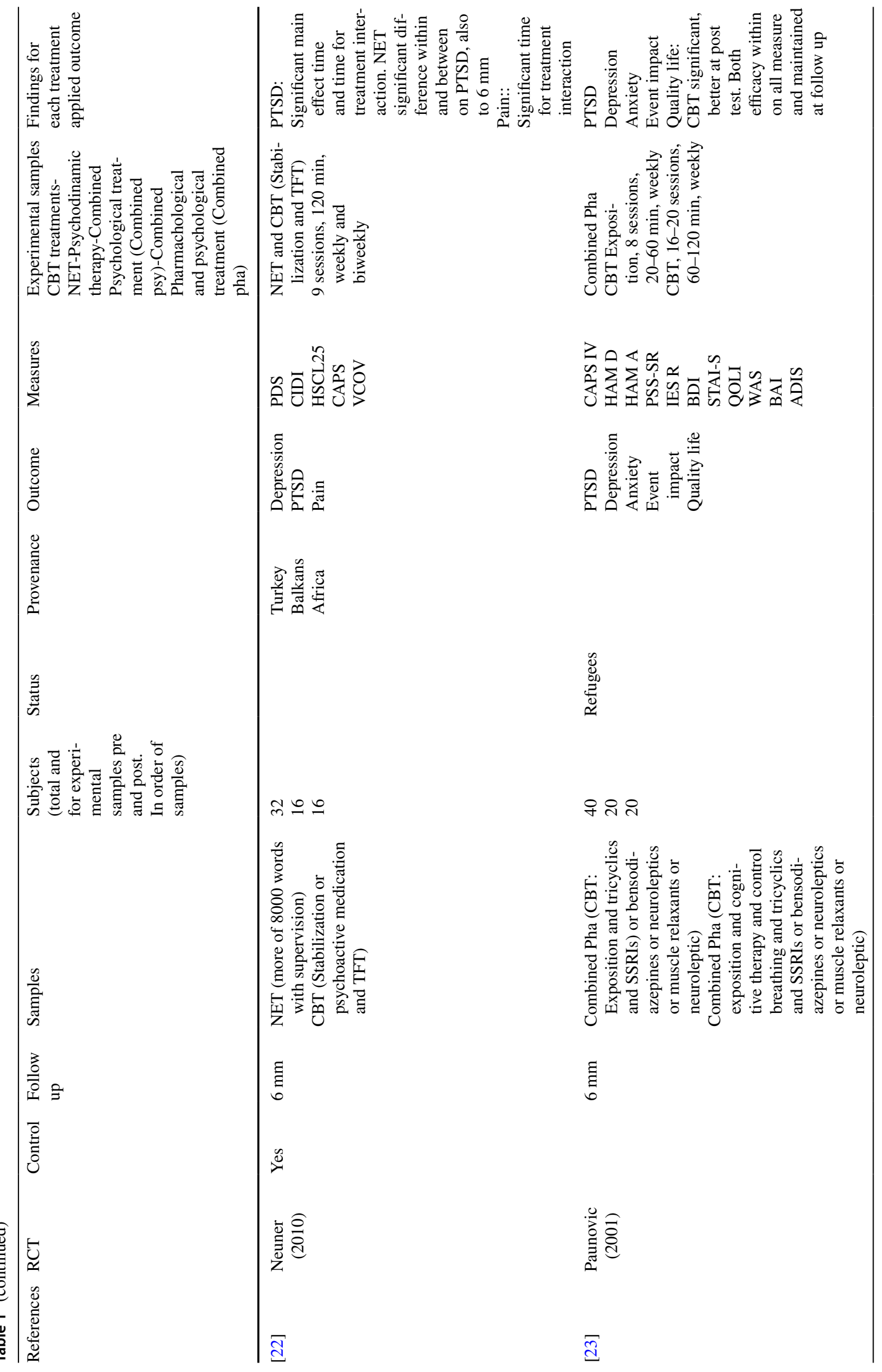




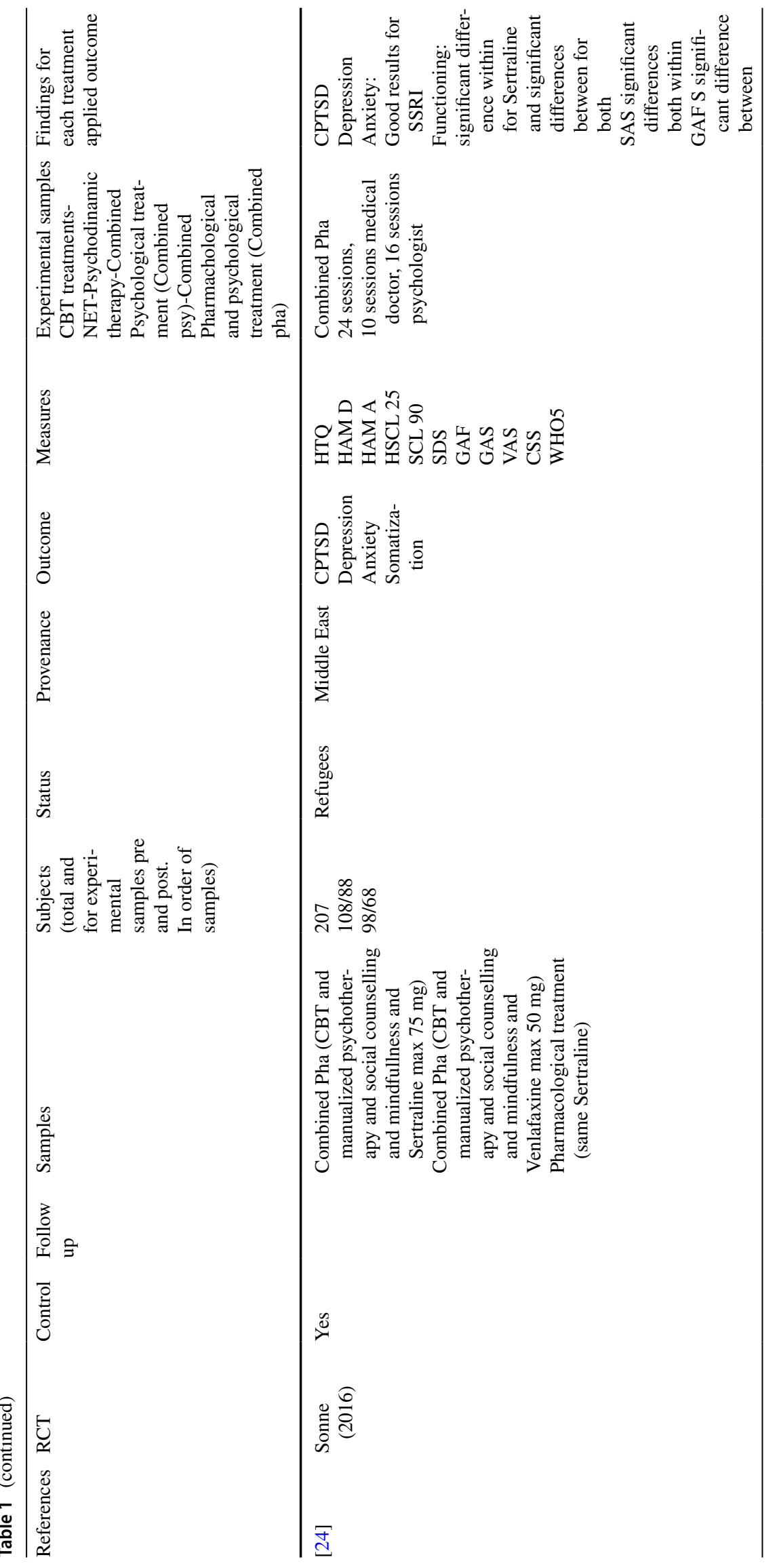




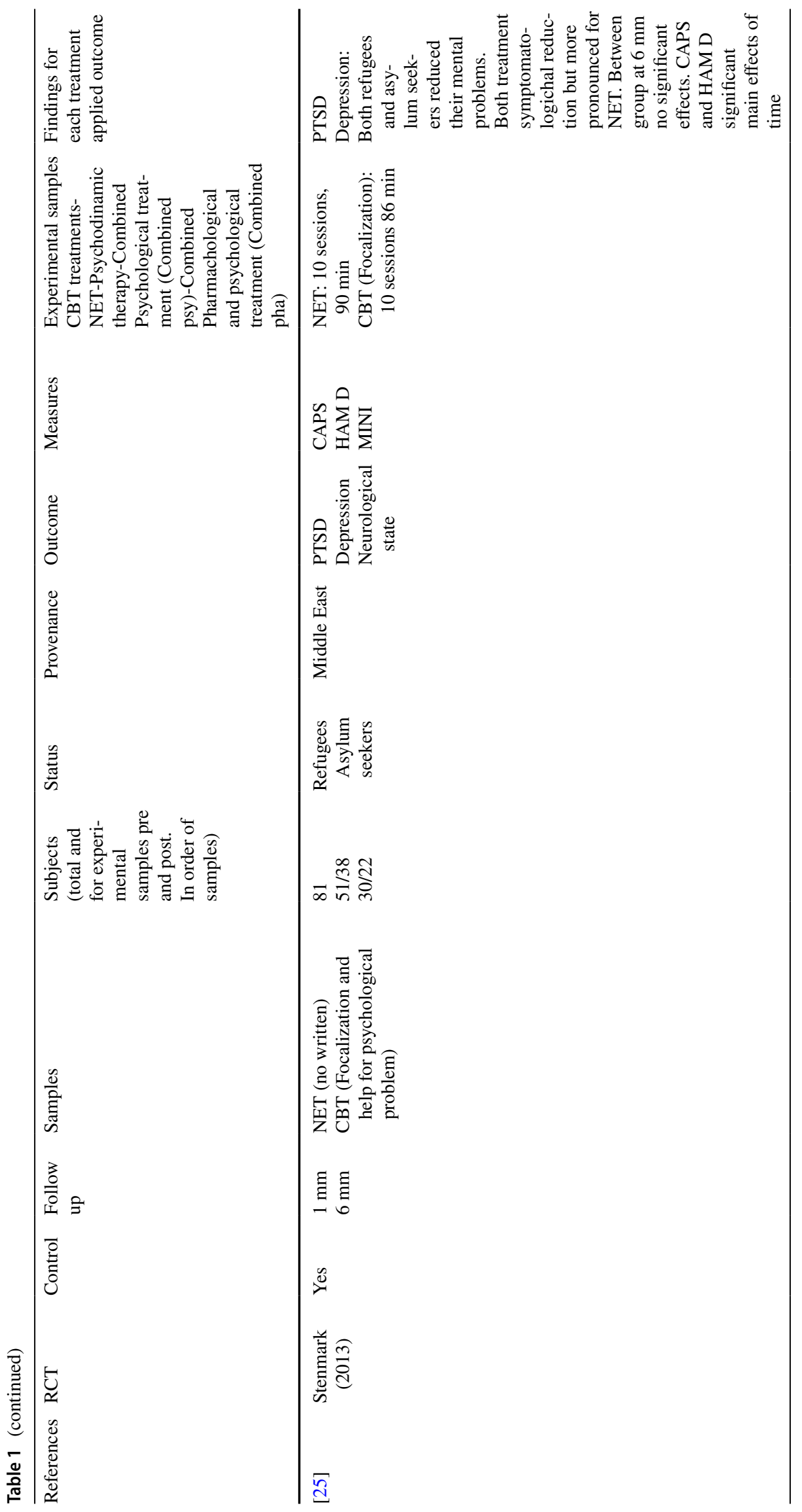




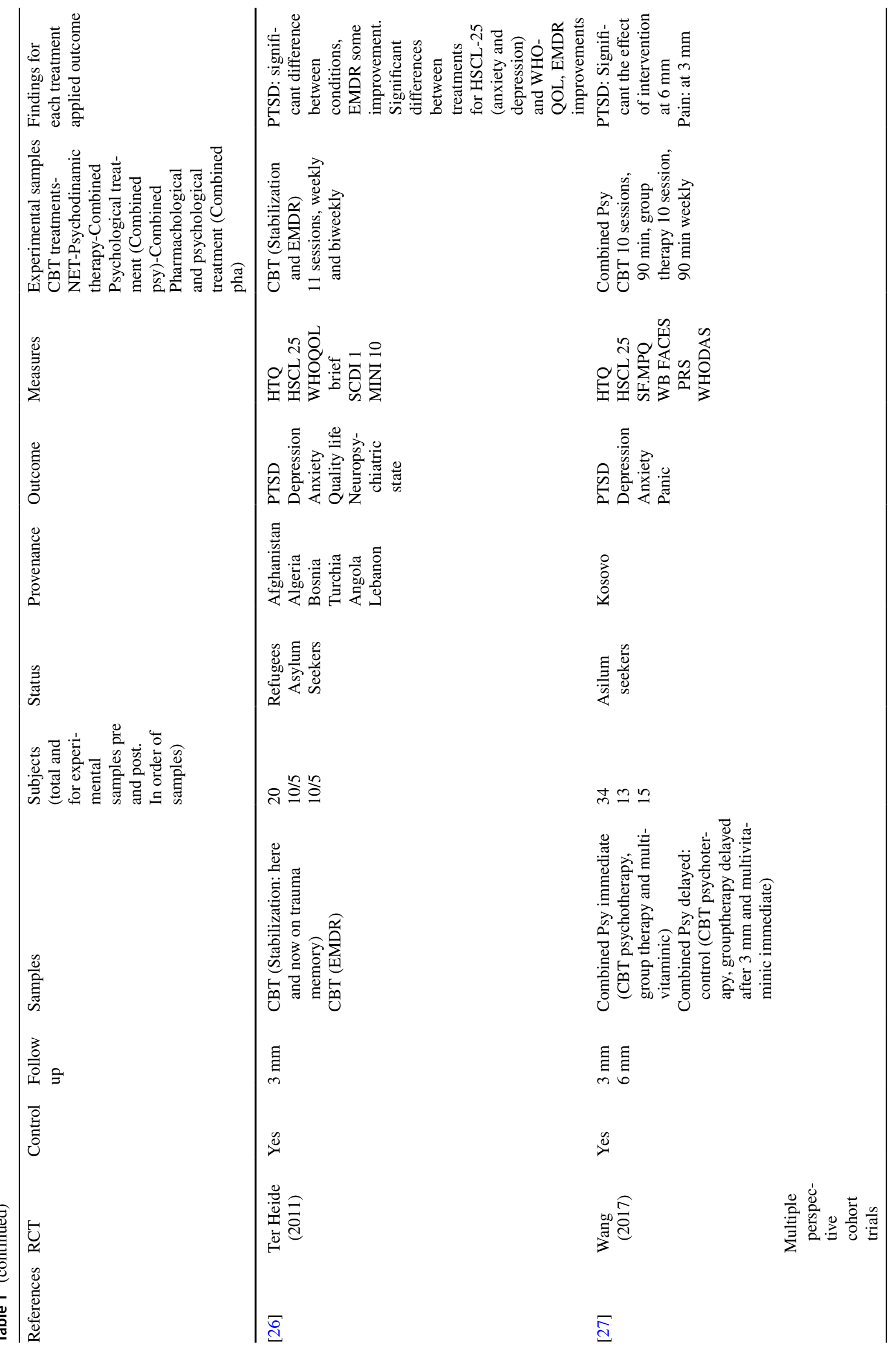




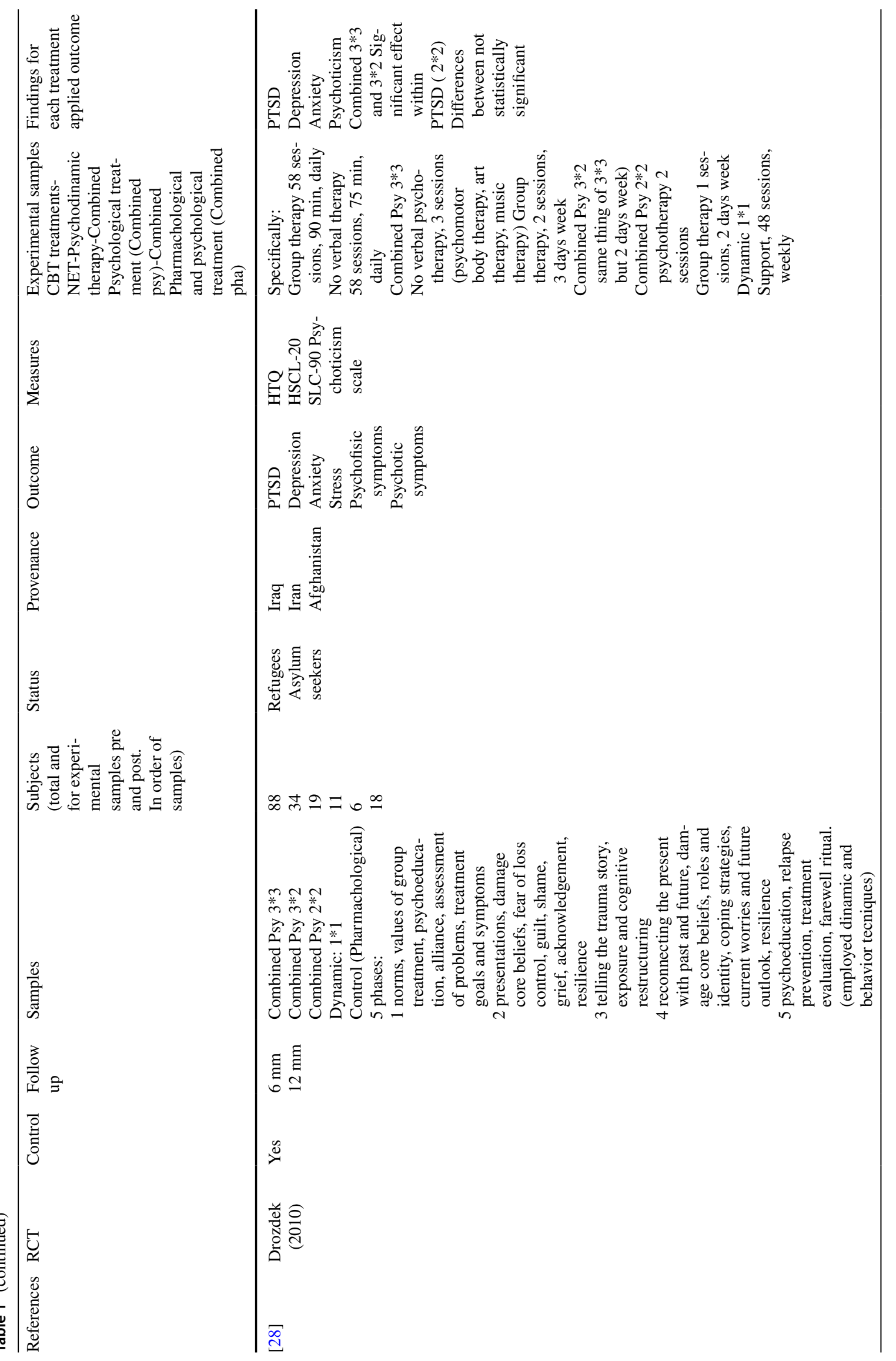




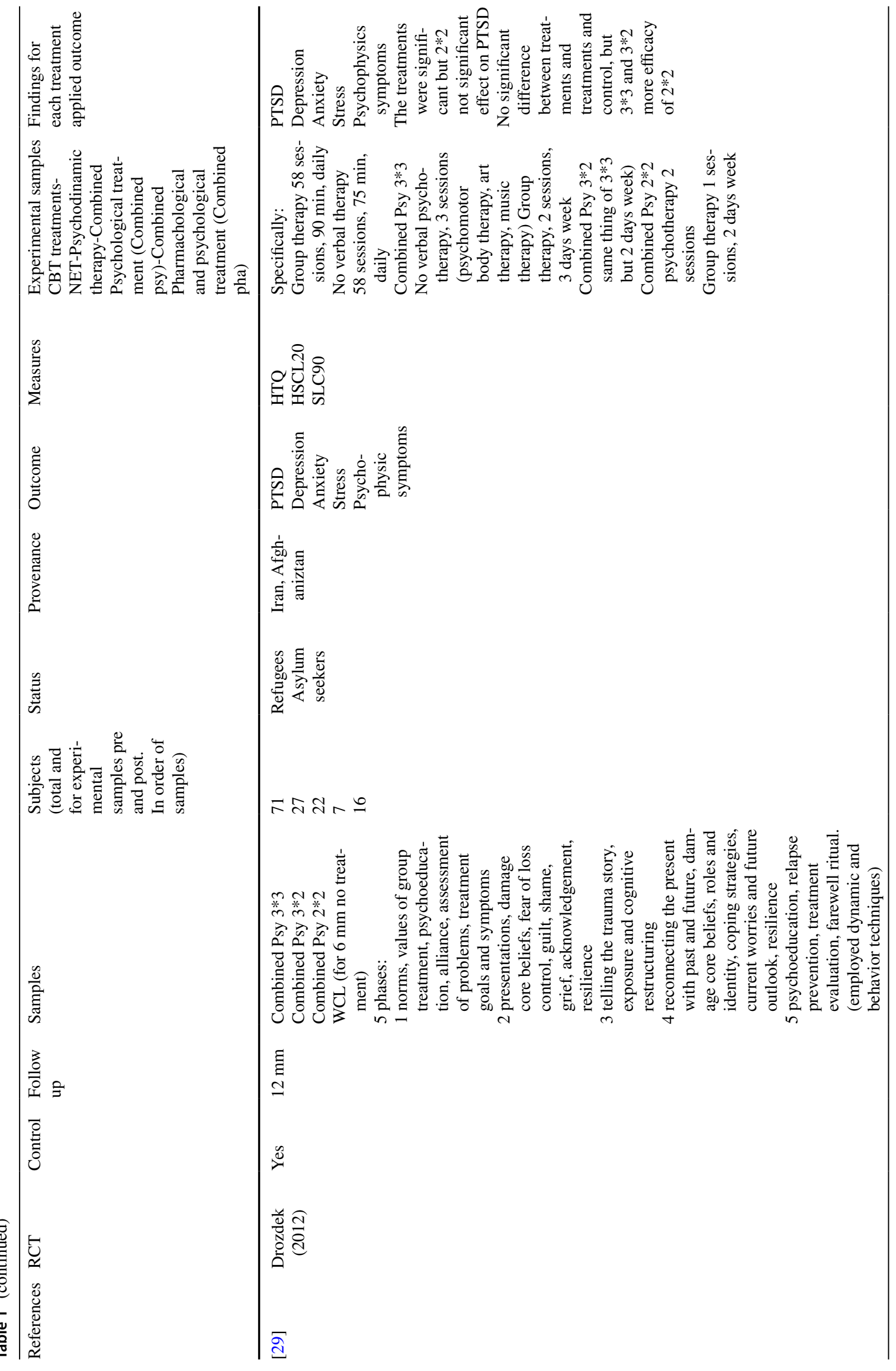




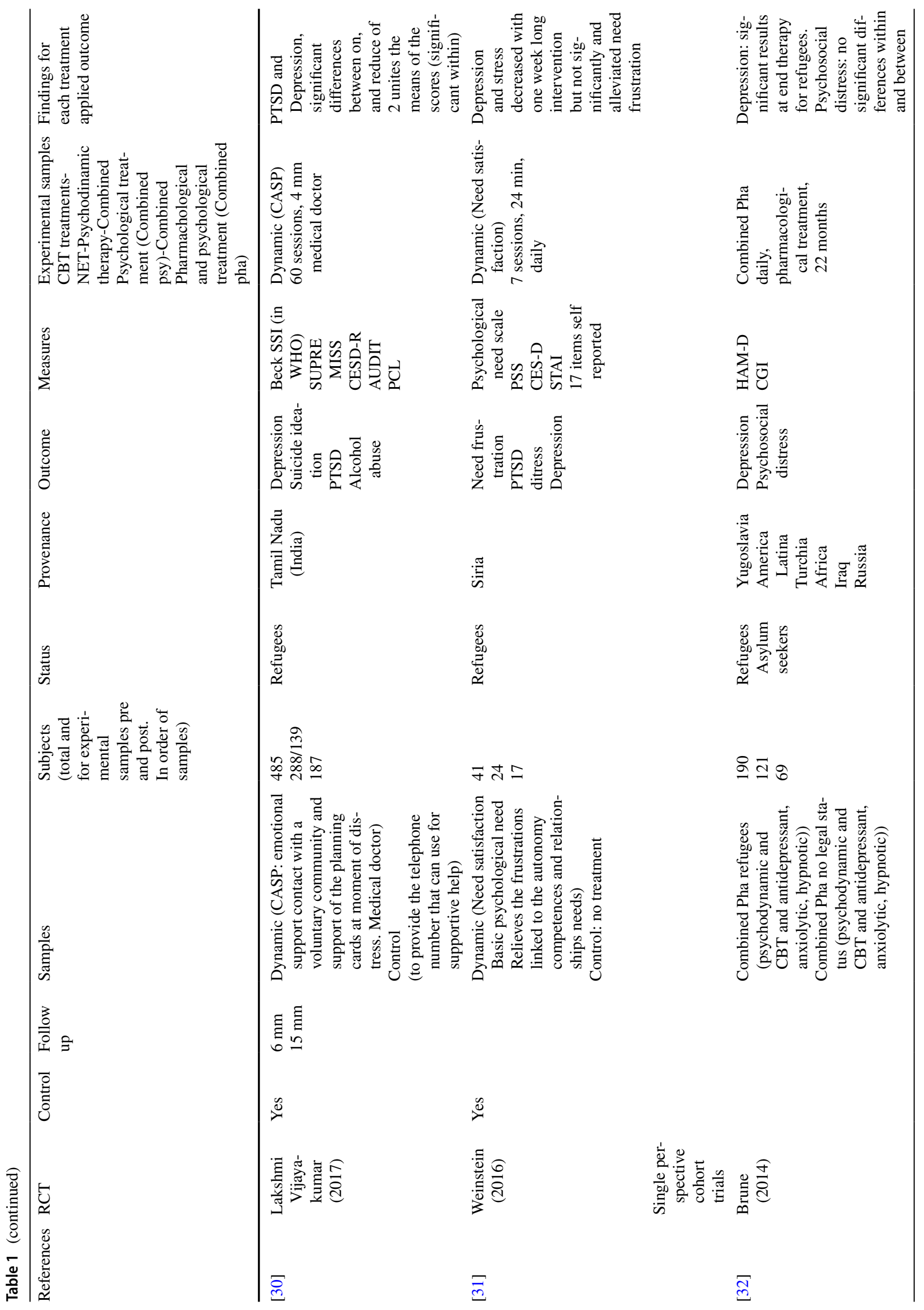




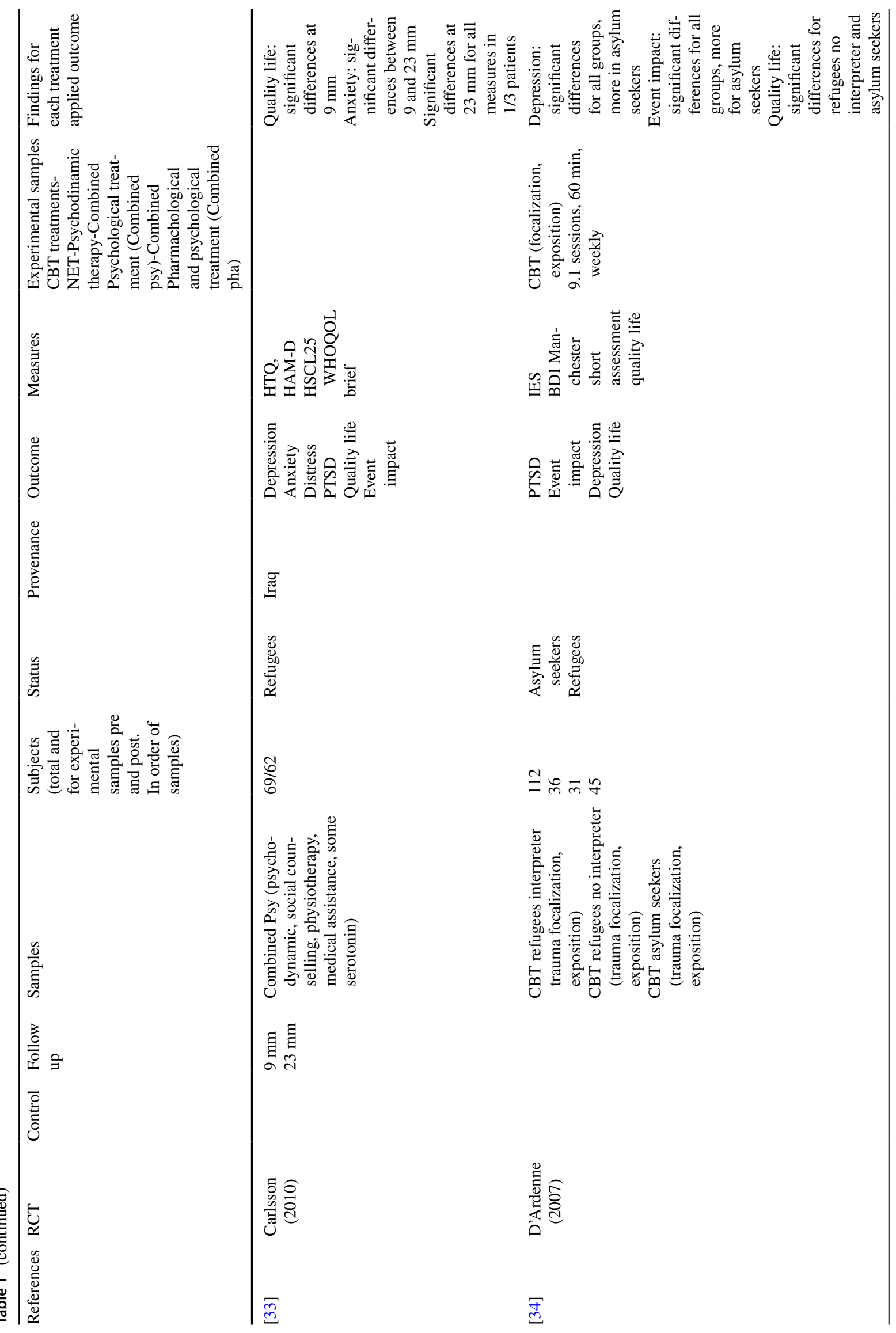




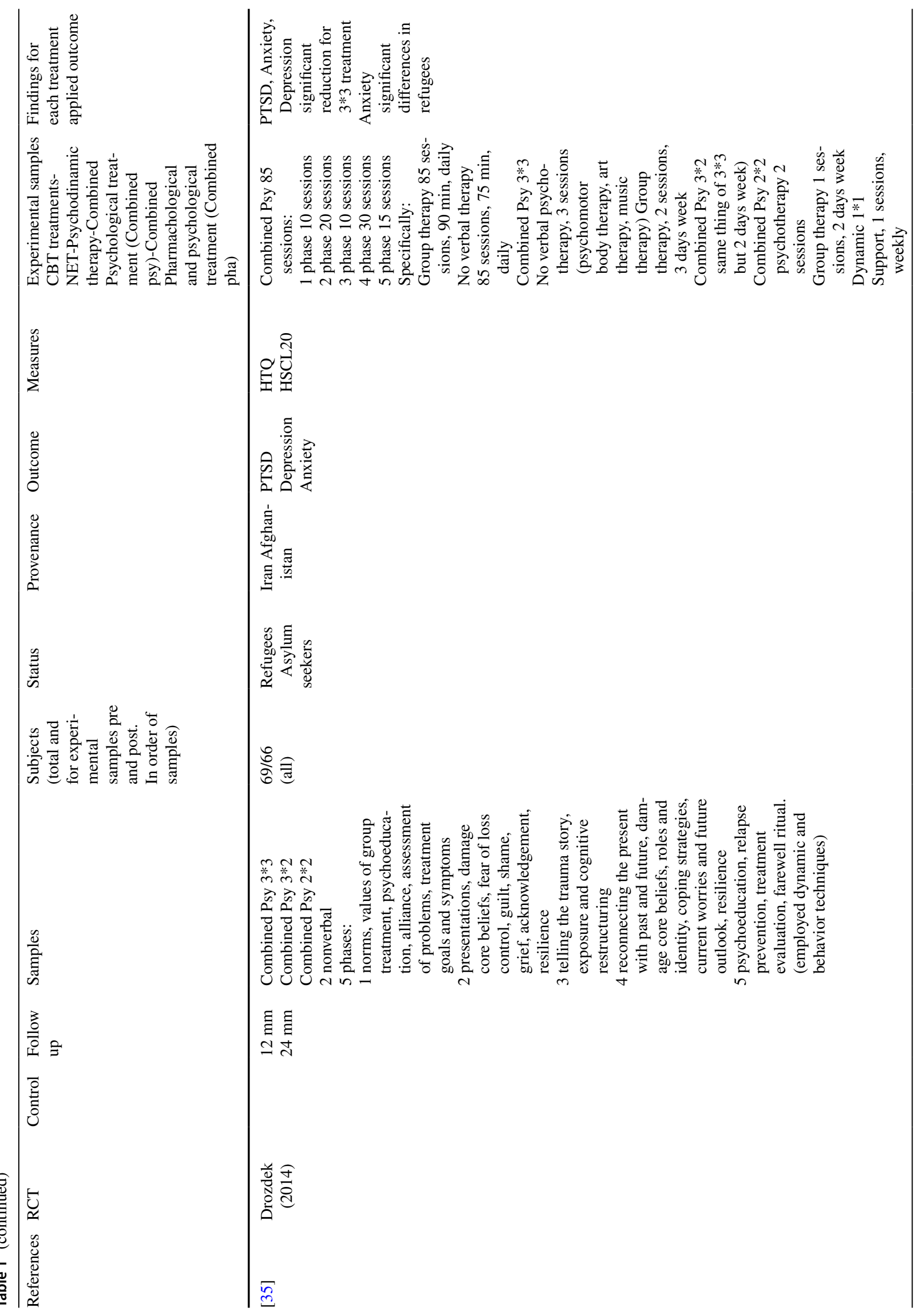




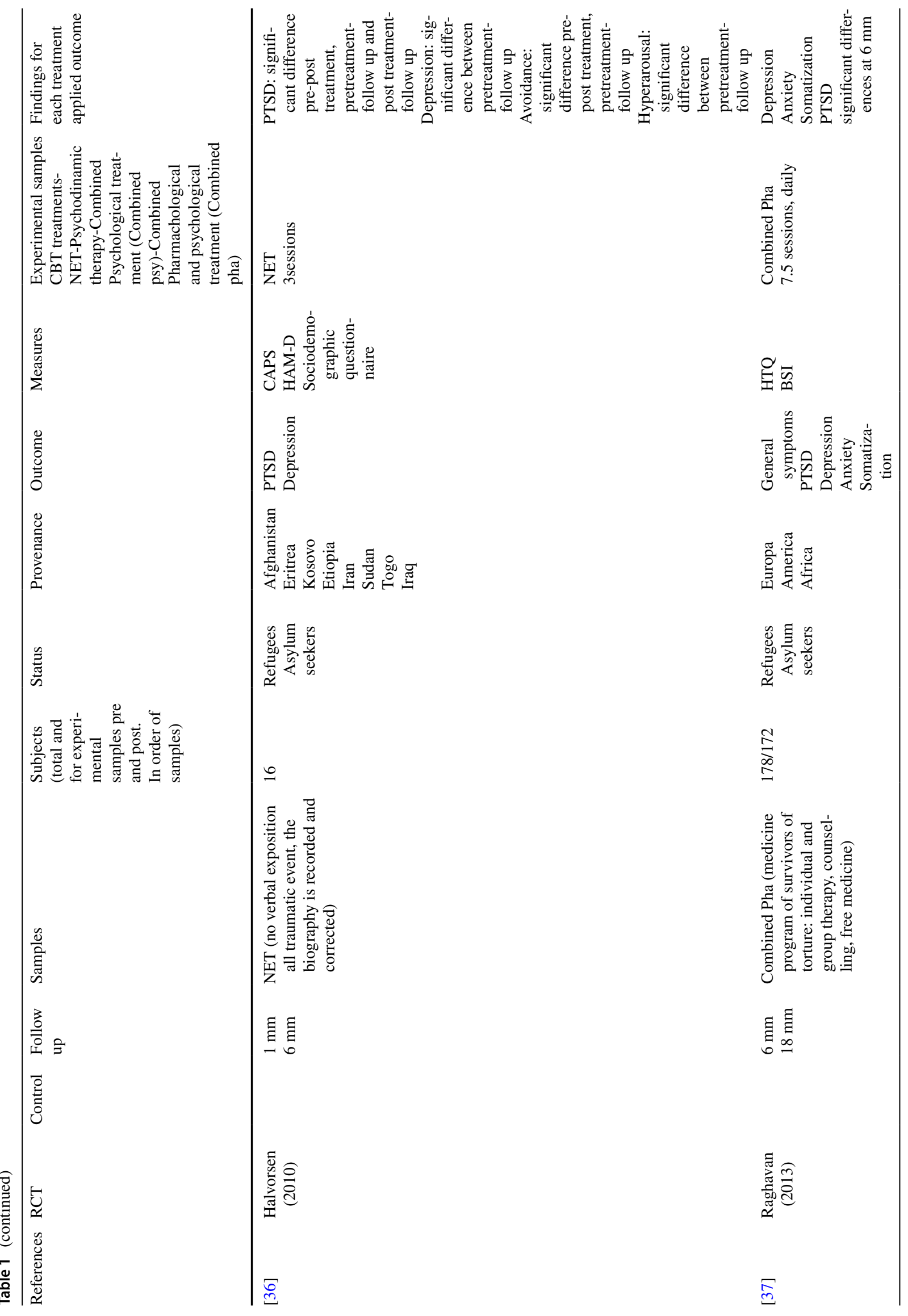




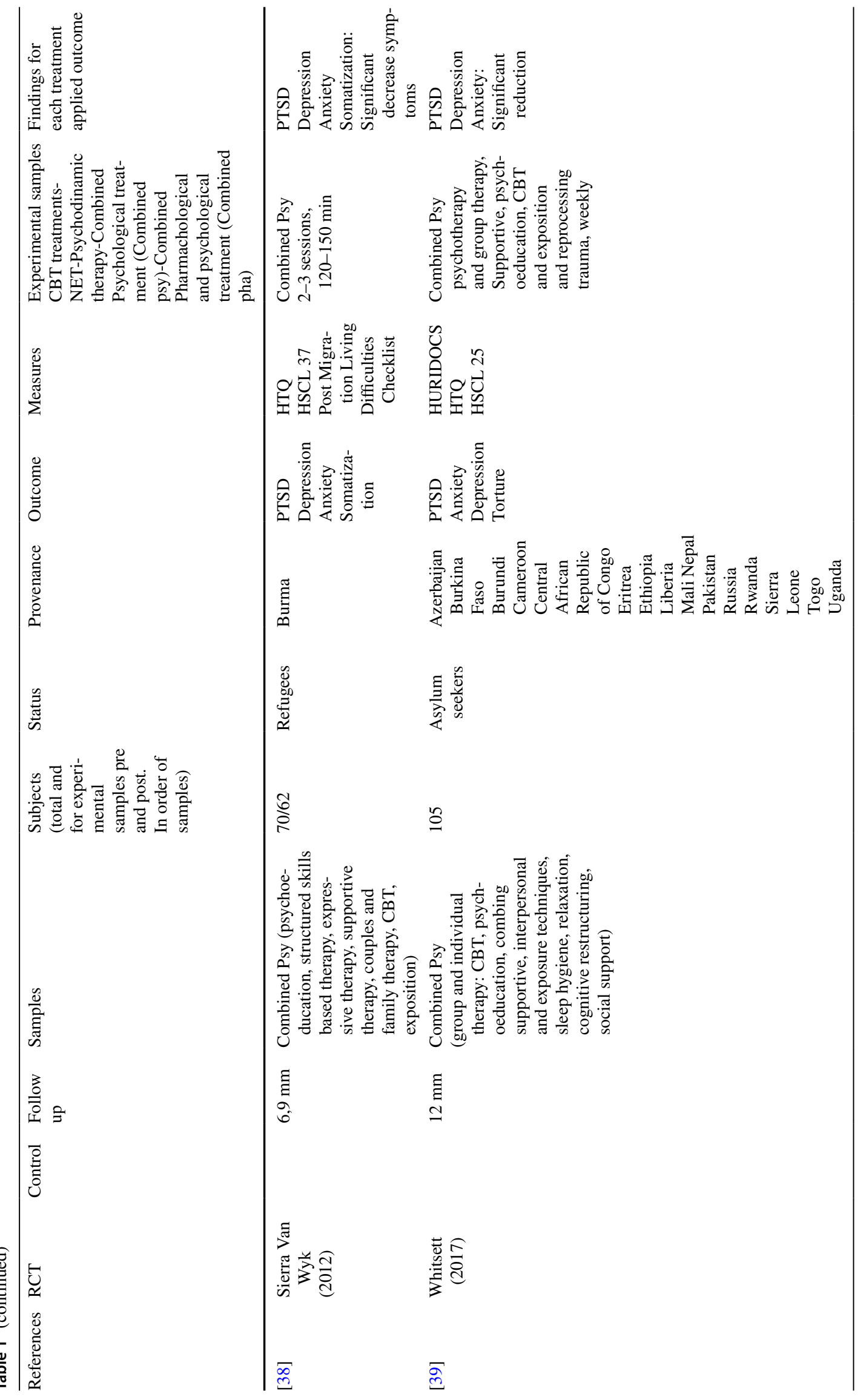




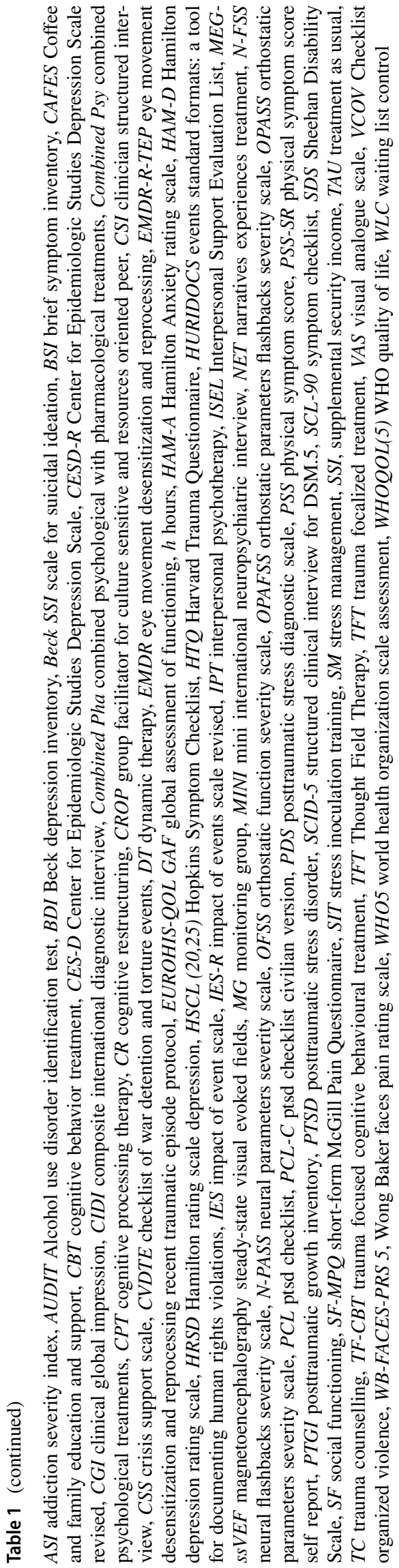

deviation, number of subjects at pre and post intervention for one of the three outcomes were provided.

\section{Metanalytic Techniques Calculation}

Mean differences (random effects model) were computed using Review Manager version 5.3. (RevMan5.3). All the mean differences were computed for depression, anxiety and somatization outcome. The program provides the following results: Heterogeneity $\left(\mathrm{Tau}^{2}, \mathrm{Chi}^{2}, \mathrm{I}^{2}\right)$ and test for overall effect (Z). Publication bias significance was evaluated by inspecting the funnel plots as implemented in RevMan5.3.

\section{Results}

\section{Study Selection}

440 research papers about migrants, refugees or asylum seeker, were included as reported in Fig. 1 (PRISMA). Afterwards the research papers on migrants receiving psychological or/and pharmacological treatment were selected, resulting 108 studies. A more specific full view paper investigation on this subsample of studies, identified only the research paper on migrants with a psychological and/or pharmacological treatment with pre and post evaluation of depression and/or anxiety and/or somatization, resulting 52 studies (1997-2018). These fifty-two studies were included in the systematic review (Supplementary material). Of these 52 studies, 25 were excluded from the metanalysis due to lacking data. Finally, 27 studies (2004-2018) were included for the metanalysis (Review Manager 5.3 program was set for calculating mean differences, standard deviation and number of subjects through a random effects model.

\section{Excluded Studies}

332 on 440 studies were excluded due to the lack of clinical intervention. Of the resulting 108 studies, 56 studies were excluded due to the following reasons: 2 studies were wrote in German language; 1 study was performed on a sample of military personnel; 1 study was conducted on a sample of interpreters; 5 studies were performed on patients who were treated in own country; 11 studies were conducted on patients with multiple trauma; 10 studies were reviews; 14 studies did not present a pre-intervention evaluation; 1 study was conducted on a single case; 11 studies were conducted on an underage sample. Of the resulting 52 studies, 25 studies did not report useful data and the authors did not answer to an email request of data or their email address was not available. 


\section{Systematic Review}

Qualitative analysis of the systematic review included 52 studies, 4 studies with control group, 21 studies with a follow up, 21 studies with both follow up and control group, 6 studies without control group and follow up.

Totally, in the 52 studies there were 4720 patients with a pre-treatment evaluation and 3913 with a pre and post treatment evaluations.

Eighty-eight trials, from the 52 studies, provided one of the following treatments: 22 trials performed the CBT (classic CBT treatments, eye movement desensitization reprocessing-EMDR, stress inoculation training-SIT, trauma counselling-TC, thought field therapy-TFT, exposition, stabilization, focalization, cognitive rebuilding-CR, self-management-SM), 9 trials performed the NET (wrote or no wrote), 15 trials performed the dynamic therapies (interpersonal psychotherapy-IPT, group facilitator for culture sensitive and resources oriented peer-CROP, Coffee and family education and support-CAFES, contact and safety planning-CSP, tea and family education and support-TAFES, satisfaction, transcendental meditation-TM, testimony psychotherapy-TP), 20 trials performed the combined cognitive psychological treatment and 23 trials performed the combined pharmacological and psychological treatments.

About legal status category, 31 studies reported refugee samples, 4 studies reported asylum seeker samples, 13 studies reported both refugee and asylum seeker samples and 4 studies reported samples with not specified socio-politic status.

About provenance category, 20 studies treated Middle east populations, 8 studies treated African populations, 7 studies treated Asian populations, one study treated South American populations, 8 studies treated populations of different provenience, 8 studies did not specify the provenance.

Mean number of sessions for each treatment category was respectively: CBT, 13 sessions of $81 \mathrm{~min}$; NET, 7 sessions of $103 \mathrm{~min}$; dynamic therapies, 22 sessions of $105 \mathrm{~min}$; combined psychological treatments, 35 sessions of $59 \mathrm{~min}$; combined psychological with pharmacological treatments, 18 sessions of $62 \mathrm{~min}$.

In the systematic review each sample was considered and included in one, in two or in all the three outcomes (depression, anxiety and somatization) considering the pre-post effect reported in each of the 52 studies resulting in one or two or three trials.

The systematic review included 52 studies for 107 samples (139 trials). Of these 139 trials, 18 trials were excluded because of were not eligible treatments: 16 samples (16 trials) were usual treatments or wait lists and 2 samples ( 2 trials) were pharmacological treatments. The samples included in the final systematic review were 89 (121 trials). Qualitative results of the systematic review showed that the
CBT (22 samples with 28 trials) had significant effect on depression (18 trials) in 11/18 trials (61\%) and no significant effect in 7/18 trials (39\%). The CBT had significant effect on anxiety ( 8 trials) in $4 / 8$ trials (50\%) and no significant effects in $4 / 8$ trials (50\%). The CBT had significant effect on somatization ( 2 trials) in $0 / 2(0 \%)$ trials and no significant effect in $2 / 2$ trials $(100 \%)$.

The NET ( 9 samples with 9 trails) had significant effects on depression in $4 / 7$ trials (57\%) and no significant effects in $3 / 7$ trials (43\%). There were not trials using NET on the anxiety outcome. The NET had significant effect on somatization in $1 / 2$ trials $(50 \%)$ and had not significant effects in $1 / 2$ trials $(50 \%)$.

The dynamic therapies ( 15 samples with 16 trials) had significant effect on depression in 5/10 trials (50\%) and had no significant effect in $5 / 10$ trials $(50 \%)$. The dynamic therapies had significant effect on anxiety in $2 / 4$ trials $(50 \%)$ and had not significant effects in $2 / 4$ trials $(50 \%)$. The dynamic therapies had significant effect on somatization in $1 / 2$ trials (50\%) and had not significant effect in $1 / 2$ trials (50\%).

Combined psychological treatments (20 samples with 31 trials) had significant effect on depression in $6 / 13$ trials (46\%) and had not significant effect in 7/13 trials (54\%). Combined psychological treatments had significant effect on anxiety in 6/13 trials (46\%) and had not significant effect in $7 / 13$ trials (54\%). Combined psychological treatments had significant effect on somatization in $3 / 5$ trials $(60 \%)$ and had not significant effect in $2 / 5$ trials (40\%).

Combined psychological and pharmacological treatments (23 samples with 37 trials) had significant effect on depression in $8 / 13$ trials $(61 \%)$ and not significant effect in $5 / 13$ trials (39\%). Combined psychological and pharmacological treatment had significant effect on anxiety in $4 / 11$ trials (36\%) and had not significant effect in $7 / 11$ trials (64\%). Combined psychological and pharmacological treatments had significant effect on somatization in 5/13 trials (39\%) and had not significant effect in $8 / 13$ trials (61\%).

\section{Metanalysis}

\section{Included Studies Characteristics and Outcome}

Metanalysis included 27 studies for 75 trials.

15 RCT, 4 of which presented a follow up, 2 showed a control sample, and 9 reported a follow up and control sample.

$4 \mathrm{MCT}$, one of which reported a control sample and 3 showed follow up and control sample.

8 SCT, 6 of which were planned with follow up, and 2 without a control sample and/or a follow up.

Depression outcome was treated in 26 studies (45 trials): 14 studies were RCT with 26 trials, 4 studies were MCT with 8 trials, and 8 studies were SCT with 11 trials. 
The anxiety outcome was treated in 14 studies ( 25 trials): 7 studies were RCT with 14 trials, 2 studies were MCT with 6 trials, and 5 studies were SCT with 5 trials.

The somatization outcome was treated in 3 RCT ( 5 trials).

Moreover, regarding RCT, 6 studies assessed depression, 6 studies investigated depression and somatization, 1 study examined depression, anxiety and somatization, 1 study assessed depression and somatization, and 1 study considered only somatization. With regard to MCT, 2 studies assessed depression and 2 studies examined depression and anxiety. As regard SCT, 5 studies investigated depression and anxiety and 3 studies considered depression.

As regard the treatment areas for RCT, 7 studies tested CBT treatments, 5 studies analyzed NET, 0 studies assessed dynamic therapies, 1 study investigated combined psychological treatments, and 5 studies examined combined psychological and pharmacological treatments. About treatment area for MCT, 3 studies tested dynamic therapies, and 2 combined psychological treatments. About treatment area for SCT, 1 study assessed CBT treatment, 1 study analyzed NET, 0 studies investigated dynamic therapies, 4 studies examined combined psychological treatments, and 2 studies explored combined psychological and pharmacological treatments.

Moreover, about the provenience area 11 RCT included Middle East populations and 3 studies tested populations from different provenances. About MCT, 3 studies tested Middle East populations, and one study Asian populations. About SCT, 2 studies included Middle East populations, 4 studies tested populations from different provenances, one study assessed African populations, and 2 studies did not specify the provenance of the participants.

Moreover, about the status area, 9 RCT investigated refugees, 2 studies included asylum seekers, 3 studies examined both refugees and asylum seekers, and one study did not specify the status of the migrant participants. As regard MCT, 2 studies included refugees, and 2 studies considered both refugees and asylum seekers. For SCT, 2 studies included refugees, one studies investigated asylum seekers, and 5 studies assessed both refugees and asylum seekers.

\section{Participants of Metanalysis}

The 27 selected studies involved the following number of participants (for all outcome): 956 participants pre-treatment and 774 participants post treatment as regard RCT design; 432 participants pre-treatment and 283 participants post treatment for MCT design; 809 participants pre-treatment and 785 participants post treatment regarding SCT design. The participants differentiated for outcome were: depression 20,177 pre-treatment and 1822 post treatment; anxiety 1225 pre-treatment and 1086 post treatment; somatization 207 pre-treatment and 184 post treatment.
The participants were adult men and women, the age range was 19-51 years for RCT, 18-70 years for MCT, and 18-80 years for SCT.

\section{Interventions Comparation of Metanalysis}

The mean number of sessions was: 9.57 sessions of $98.7 \mathrm{~min}$ as regard RCT for CBT; 8.8 sessions of 96.6 min for NET; 10 sessions of $90 \mathrm{~min}$ for combined psychological treatments; 11.22 sessions of 70 min for combined pharmacological and psychological treatments. As regard MCT there were found 60 sessions of $24 \mathrm{~min}$ for dynamic therapies and 71.5 sessions of $82.5 \mathrm{~min}$ for combined psychological treatments. With regard to SCT there were found 9.1 sessions of $60 \mathrm{~min}$ for CBT; 3 sessions for NET; 22 sessions of $23.25 \mathrm{~min}$ for combined psychological treatments; 47.75 sessions for combined pharmacological and psychological treatments.

\section{Comparators}

Comparators included the experimental samples and control samples if treated with CBT, NET, dynamic therapies with different manualization or combined psychological treatments and combined psychological and pharmacological treatments. There have been excluded the control samples as waiting lists.

\section{Treatments Effects: Effect Size About Metanalysis}

Metanalytic analysis was performed on 27 selected studies separating the studies for outcome, depression, anxiety and somatization and for each different study design (RCT, MCT, and SCT). Successively, because of the high heterogeneity, further sub-metanalysis have been performed following the areas of interest (at least 4 trials were sufficient to perform a metanalysis): socio-politic status (asylum seekers and refugees), provenance, and treatment's kind. The sub-metanalysis were performed considering the different outcomes and the different research designs.

\section{Depression Outcome}

3 main forest plots ( 7 forest plots, 2 funnel plots inside Supplementary Figures) have been obtained.

RCT-depression (Fig. 2): 936-754 participants prepost treatment; Heterogeneity: $\mathrm{Tau}^{2}$ 2.3, $\mathrm{Chi}^{2}$ 803.72, df 25 ( $\mathrm{p}<0.00001), \mathrm{I}^{2} 97 \%$; test for overall effect: Z 8.93 $(\mathrm{p}<0.00001)$.

MCT-depression: 432-283 participants pre-post treatment; heterogeneity: $\mathrm{Tau}^{2} 0.08, \mathrm{Chi}^{2} 25.02$, df $7(\mathrm{p}=0.0008)$, $\mathrm{I}^{2} 72 \%$; test for overall effect: Z $4.08(\mathrm{p}<0.0001)$. 
Fig. 1 Flow chart selection and organization of the inclusion criteria and the studies for outcome (depression, anxiety, somatization) and research design (randomized controlled trial, multiple cohort trial, single cohort trial)

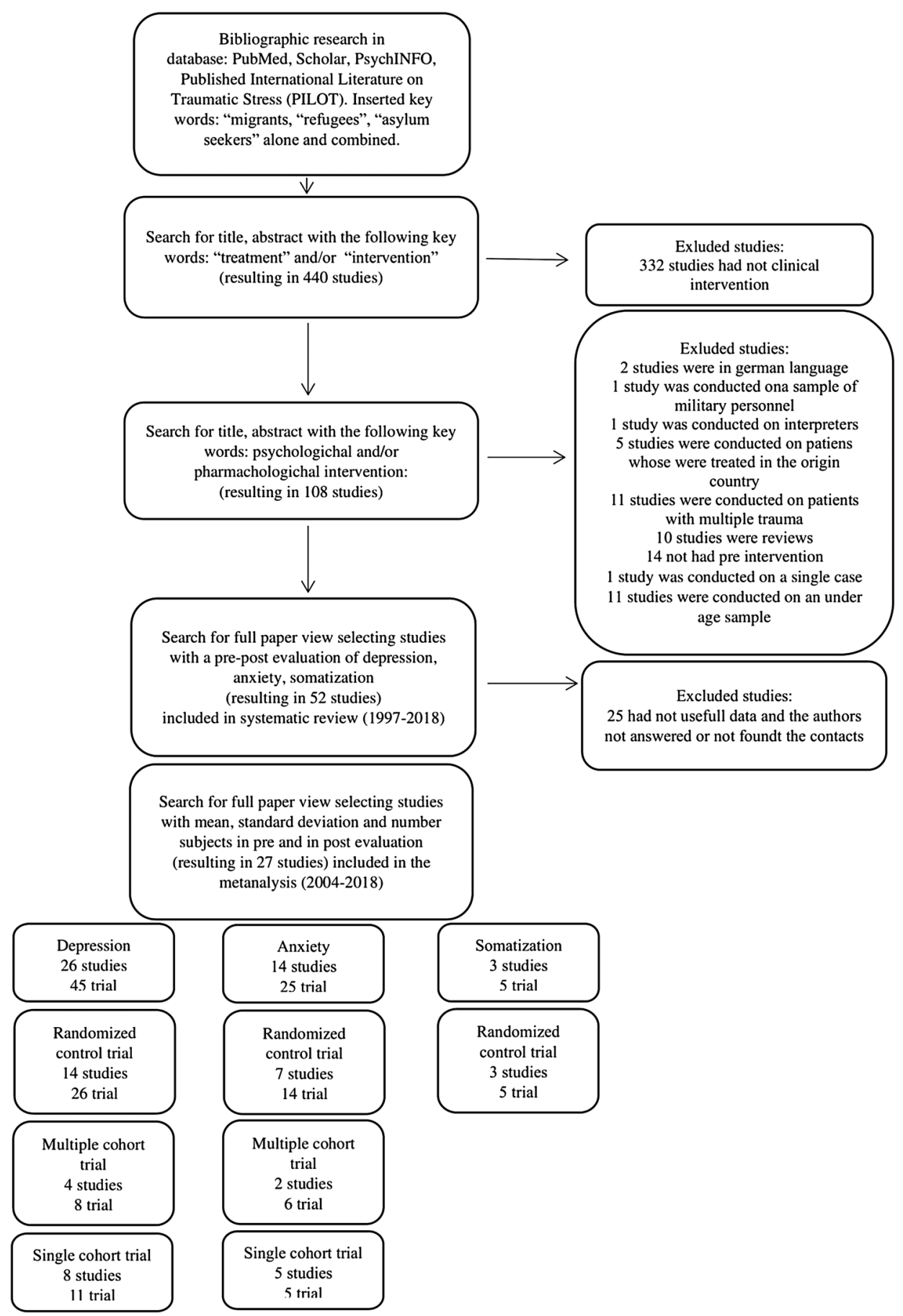

SCT-depression: 809-785 participants pre-post treatment; Heterogeneity: $\mathrm{Tau}^{2}$ 0.56, $\mathrm{Chi}^{2} 269.25$, df 10 (p<0.00001), $\mathrm{I}^{2} 96 \%$; test for overall effect: Z 6.45 ( $\left.\mathrm{p}<0.00001\right)$.

\section{Sub-metanalysis Results on the Kind of Treatment for Depression RCT}

CBT: 213-162 participants pre-post treatment (7 studies-8 trial). Heterogeneity: $\mathrm{Tau}^{2} 5.15 ; \mathrm{Chi}^{2} 230.88, \mathrm{df}=7$, $\mathrm{p}<0.00001 ; \mathrm{I}^{2}$ 97\%; test for overall effect: $\mathrm{Z} 4.02 \mathrm{p}=0.0001$.

NET: $138-114$ participants pre-post treatment (5 studies-5 trial). Heterogeneity: $\mathrm{Tau}^{2} 23.22$; $\mathrm{Chi}^{2} 37.02 \mathrm{df}=4$ $\mathrm{p}<0.00001 ; \mathrm{I}^{2} 89 \%$; test for overall effect: $\mathrm{Z} 2.4 \mathrm{p}=0.02$. 


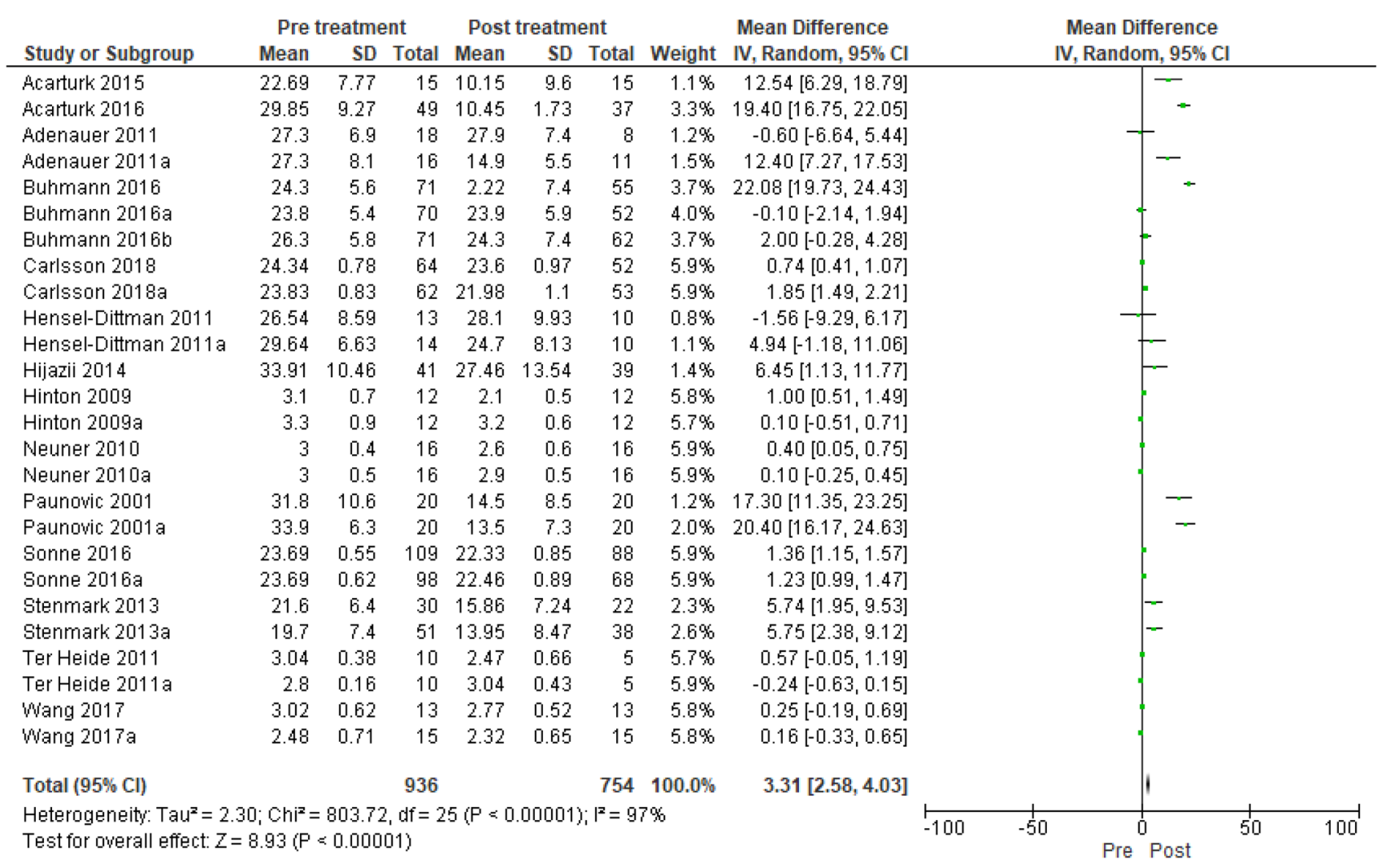

Fig. 2 Forest plot that describes the difference pre and post intervention in the depression outcome. RCT research design

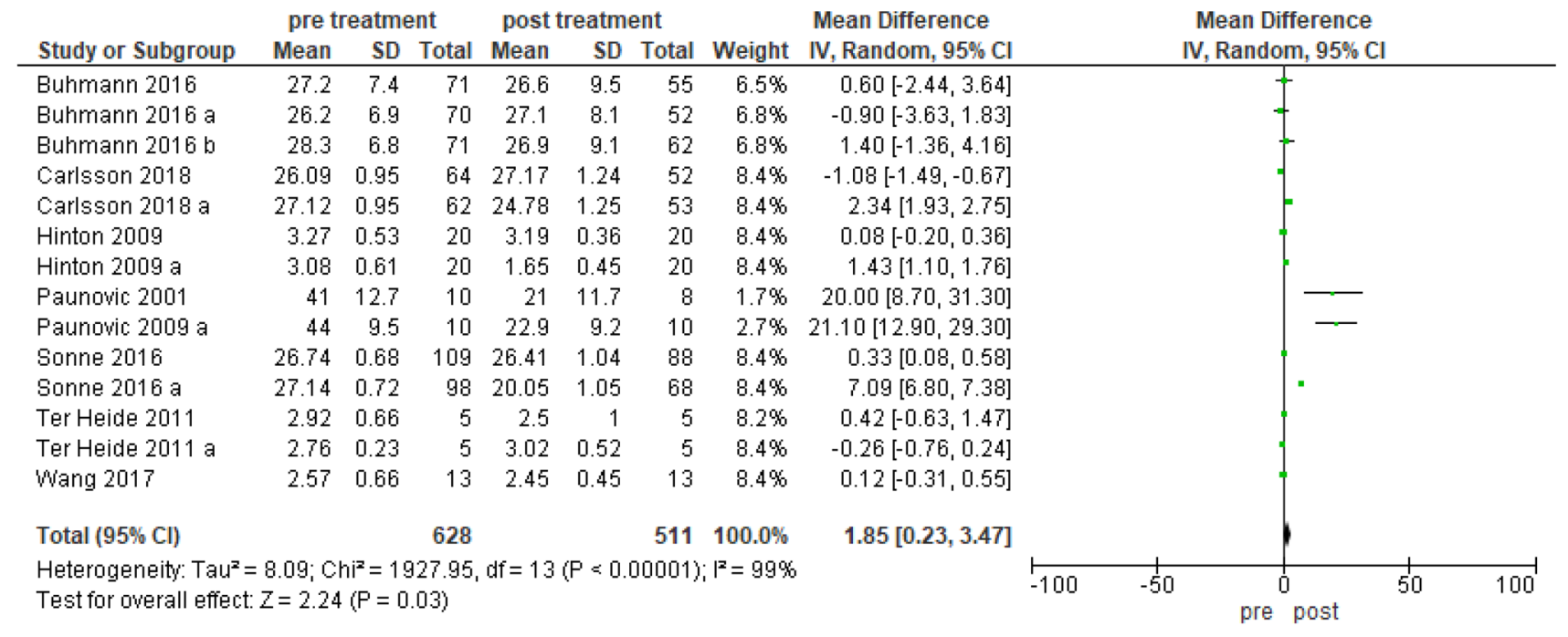

Fig. 3 Forest plot that describes the difference pre and post intervention in the anxiety outcome. RCT research design

Combined psychological and pharmacological treatments: 539-442 participants pre-post treatment (5 studies-10 trial). Heterogeneity: $\mathrm{Tau}^{2}$ 2.30; $\mathrm{Chi}^{2} 444.14$ $\mathrm{df}=9 \mathrm{p}<0.00001 ; \mathrm{I}^{2} 98 \%$; test for overall effect: Z 7.44 $\mathrm{p}<0.00001$.

\section{Sub-metanalysis Results on the Kind of Status for Depression RCT}

Refugees: $714-585$ participants pre-post treatment (8 studies-14 trial). Heterogeneity: $\mathrm{Tau}^{2}$ 3.28; $\mathrm{Chi}^{2} 640.06$ 


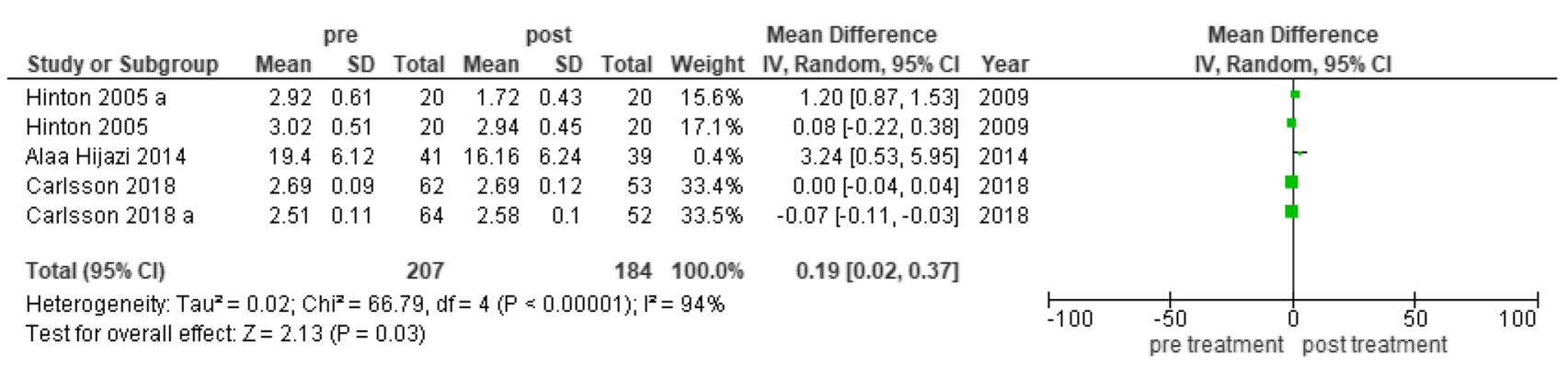

Fig. 4 Forest plot that describes the difference pre and post intervention in the somatization outcome. RCT research design

df $=13$ p $<0.00001 ; I^{2} 98 \%$; Test for overall effect: Z 9.34 $\mathrm{p}<0.00001$.

Asylum seekers: $87-80$ participants pre-post treatment (3 studies -6 trial). Heterogeneity: $\mathrm{Tau}^{2} 0.00 ; \mathrm{Chi}^{2} 3.99 \mathrm{df}=5$ $\mathrm{p}<0.55 ; \mathrm{I}^{2} 0 \%$; test for overall effect: Z $2.36 \mathrm{p}<0.02$.

\section{Sub-metanalysis Results for Kind of Status for Depression SCT}

Refugees: $327-312$ participants pre-post treatment (4 studies -5 trial). Heterogeneity: $\mathrm{Tau}^{2} 1.40 ; \mathrm{Chi}^{2} 143.98$ $\mathrm{df}=4 \mathrm{p}<0.00001 ; \mathrm{I}^{2} 97 \%$; Test for overall effect: $\mathrm{Z} 4.03$ $\mathrm{p}<0.0001$.

\section{Sub-metanalysis Results on the Kind of Provenance for Depression RCT}

Middle East: 837-662 participants pre-post treatment (11 studies-20 trial). Heterogeneity: $\mathrm{Tau}^{2} 2.34 ; \mathrm{Chi}^{2} 654.76$ $\mathrm{df}=19 \mathrm{p}<0.00001 ; \mathrm{I}^{2} 97 \%$; test for overall effect: Z 7.75 $\mathrm{p}<0.00001$.

\section{Sub-metanalysis Results for Kind of Treatment for Depression SCT}

Combined psychological treatment: 313-295 participants pre-post treatment (4 studies-4 trial). Heterogeneity: $\mathrm{Tau}^{2}$ 0.04; $\mathrm{Chi}^{2} 15.59 \mathrm{df}=3 \mathrm{p}=0.001 ; \mathrm{I}^{2} 81 \%$; Test for overall effect: Z $4.08 \mathrm{p}<0.0001$.

\section{Anxiety Outcome}

RCT-anxiety (Fig. 3): 628-511 participants pre-post treatment; heterogeneity: $\mathrm{Tau}^{2} 8.09, \mathrm{Chi}^{2} 1927.95 \mathrm{df}$ 13 ( $p<0.00001), \mathrm{I}^{2} 99 \%$; test for overall effect: Z 2.24 $(\mathrm{p}=0.03)$.

MCT-anxiety: 108 participants pre e post treatment; heterogeneity: $\mathrm{Tau}^{2}$ 0.49, $\mathrm{Chi}^{2} 91.99$, df 5 ( $\left.\mathrm{p}<0.00001\right), \mathrm{I}^{2}$ 95\%; test for overall effect: Z $2.69(\mathrm{p}=0.007)$.
SCT-anxiety: 489-467 participants pre-post treatment; heterogeneity: $\mathrm{Tau}^{2} \mathrm{0} 0.06, \mathrm{Chi}^{2} 28.66 \mathrm{df} 4(\mathrm{p}=0.00001), \mathrm{I}^{2}$ $86 \%$; test for overall effect Z $3.40(p=0.0007)$.

\section{Sub-metanalysis for Kind of Status on Anxiety RCT}

Refugees: 605-488 participants pre-post treatment (5 studies-11 trial). Heterogeneity: $\mathrm{Tau}^{2}$ 9.31; $\mathrm{Chi}^{2} 1813.09$ $\mathrm{df}=10 \mathrm{p}<0.00001 ; \mathrm{I}^{2} 99 \%$; test for overall effect: Z 2.49 $\mathrm{p}=0.01$.

\section{Sub-metanalysis for Kind of Provenance on Anxiety RCT}

Middle East: $558-443$ participants pre-post treatment (4 studies -8 trial). Heterogeneity: $\mathrm{Tau}^{2}$ 12.49; $\mathrm{Chi}^{2} 1664.77$ df $=7 \mathrm{p}<0.00001 ; \mathrm{I}^{2} 100 \%$; test for overall effect: $\mathrm{Z} 1$ $\mathrm{p}=0.32$.

\section{Sub-metanalysis for Kind of Treatment on Anxiety RCT}

Combined psychological with pharmacological treatments: 535-436 participants pre-post treatment (5 studies-10 trial). Heterogeneity: $\mathrm{Tau}^{2}$ 9.32; $\mathrm{Chi}^{2} 1809.25 \mathrm{df}=9$ $\mathrm{p}<0.0001 ; \mathrm{I}^{2} 100 \%$; test for overall effect: Z $2.69 \mathrm{p}=0.007$.

\section{Somatization Outcome}

RCT-somatization (Fig. 4): 207-184 participants prepost treatment. Heterogeneity: $\mathrm{Tau}^{2} \mathrm{0.02}, \mathrm{Chi}^{2}$ 66.79, df $4(\mathrm{p}=0.00001), \mathrm{I}^{2} 94 \%$; test for overall effect: Z 2.13 $(\mathrm{p}=0.03)$.

\section{Methodological Quality: Publication Bias}

The risk of bias among studies was high for many reasons, 13/27 (metanalysis) 30/52 (systematic review) publications were MCT and SCT studies this was the first issue hampering the methodological quality of the studies. Moreover, in many studies the number of participants at the pre-treatment 
phase (T0) decreased in the post treatment (T1). Often treatments with the same approach were manualized in different ways. In some studies, the samples included both refugees and asylum seekers. The different status kind could have a different impact on the psychological health of the participants. Many studies did not have an appropriate random generation assignment sequence and did not report an occultation of the assignment sequence. Some trials coming from different studies, sometimes, used different instruments of measure to evaluate the same constructs.

Further, different types of bias were detected in the studies selected for the present metanalysis and included: bias of data base, bias of inclusion, bias of language, and bias of effect size. Moreover, some studies did not report possible risks of bias. The summary of all biases and the high heterogeneity were reported in two funnel plots. However, the two funnel plots showed a good symmetry.

\section{Discussion}

The main finding of the present metanalysis is that all treatments categories, and particularly the cognitive behavioural treatments, showed to be efficient on depression.

Moreover, the effect of the treatment was lower on the asylum seekers compared to the refugees.

Specifically, the cognitive behavioural treatments together combined psychological and pharmacological treatments seemed to be more efficacious on depression, even if in a lower number of studies.

The dynamic therapies showed a discrete efficacy trough observational methodological designs, there is a need of further investigation in this field due to the total lack of RCT.

As regard the migrant's status (refugee or asylum seeker), the efficacy of the treatment on the depression showed a significant effect only for the status of refugee, whereas the effect was at the limit of significance with null degree of heterogeneity for the state of asylum seeker. This difference be could be due to adherence to the institutional expectation common in the asylum seekers applicants. In facts, they may have distorted the answers to the questionnaires in order to support or to influence the acceptation of their instance to receive an international protection [40]. This social desirability bias could modulate the pre-post evaluation differences or to attenuate the efficacy of the psychological intervention. This finding suggests to consider with great attention the variable of the status, and overall the pending instance condition of the migrant participants in the future studies. Considering that UNHCR data in Italy reported that in the first half of 2017 the total number of applications examined amounted to 41,379 ; only 4.3 out of 10 had a positive outcome (refugee status: $9 \%$; subsidiary protection: 9.8\%; permit for humanitarian reasons: $24.5 \%$ ); for $51.7 \%$ the exam ended with a denial, and $4.9 \%$ of the instances of the applicants were lost [41].

Other important results were that the psychological interventions showed an effect on the mental health of migrants. The more evident effect of the psychological treatments was on the depression, despite the systematic review showed that only the $50 \%$ of the RCT found a significant difference between the pre and post evaluation. The specific metanalysis and sub-metanalysis showed high significance levels. For all the types of treatments with the exception of the narrative exposure therapies, that showed effects at the limit of significance. This finding suggest to conduct further experimental studies in order to confirm the efficacy of narrative exposure therapies on the depression outcome. Moreover the combined (psychological and pharmacological/psychological) treatments seem to show increased benefits compared to the single ones.

The metanalysis on depression in not randomized studies (multiple and single perspective design studies) showed high levels of significance but with high levels of the heterogeneity.

Differently from the depression, the anxiety showed smaller effects of the psychological treatment. Not only the metanalysis showed decreased pre-post treatment effects on anxiety, but also the qualitative observation on the systematic review showed a probability lower than $50 \%$ to have a significant pre-post treatment effects on trials with anxiety. Moreover, the number of the studies criteria allowed to perform only the metanalysis on the RCT studies with the combined psychological/pharmacological treatments where the effect was statistically significant. It seems necessary to plane further randomized studies in order to test the efficacy of specific psychological treatments on outcomes other than depression.

Moreover, the metanalysis performed only in middleeast migrant population showed a clear statistical pre-post effect of the psychological treatment on the depression outcome but not on the anxiety outcome. This result could be explained with the cultural characteristics of these populations where the expression of the anxiety is under-represented compared to other cultures [42].

Another important finding of the present study was that, at today, there are few studies on African and Asian populations compared to the Middle East population. In the last years, a large entrance in Europe of the African population, as well as Syrian and Iranian population, has been observed. It seems to be necessary to increase the trials on the participants who have been involved in the current phenomena of migration as African, Asian, Syrian, and Iranian populations.

As regard the somatization outcome, the qualitative observation from the systematic review showed a probability of $42 \%$ to have a significant pre-post treatment effects on 
randomized control, multiple and single perspective cohort trials of $42 \%$. This limited qualitative evidence was confirmed by the metanalyses on the RCT where the statistical effect was at the limit of significance. This finding suggests the need to conduct more studies focused on testing the efficacy of psychological treatments on somatization, talking into consideration of the population at today involved in the migration phenomena characterized by a culture where somatization could represent a privileged channel to express their psychological disease [43].

Moreover, the limited number of studies with outcome of anxiety and somatization did not allow to compare the efficacy of different types of psychological treatment on these outcomes revealing a hole in the literature that it is necessary fil up in the next future.

\section{Future Research}

For future developments, we suggest to extend the research to the outcome of psychological support for the migrants using a dynamic approach, possibly through RCT design. Moreover, it seems to necessary to confirm the efficacy of narrative exposure therapy on the depression increasing the studies number on this issue. According to this line, it could be interesting to turn the attention also to the expressive writing treatment of Pennebaker [44]. This treatment shows is more systematized procedure compared to the other narrative exposure therapies and it seems to demonstrate a good efficacy in brief times (3-5 days) adopting an exclusively written application modality. Nowadays, this type of treatment was not tested on migrants yet.

Finally, it would be useful increases the number of studies of all psychological treatments applied on anxiety and somatization, possibly through RCT design.

\section{Limitations}

Few studies among those included in this systematic review treated the asylum seekers respect to the refugees.

Acknowledgements Open access funding provided by Università degli Studi di Roma La Sapienza within the CRUI-CARE Agreement.

Author Contributions DS drafted the protocol and with CL, PA, EB assessed the eligibility of the studies for inclusion, and extracted data. PA and DS developed the statistical code and performed the analyses. All authors contributed to the interpretation of the findings and to the drafting and editing of the manuscript.

Funding This research did not receive any specific grant from funding agencies in the public, commercial, or not-for-profit sectors.

\section{Compliance with Ethical Standards}

Conflict of interest The authors declare that they have no competing interests.

Open Access This article is licensed under a Creative Commons Attribution 4.0 International License, which permits use, sharing, adaptation, distribution and reproduction in any medium or format, as long as you give appropriate credit to the original author(s) and the source, provide a link to the Creative Commons licence, and indicate if changes were made. The images or other third party material in this article are included in the article's Creative Commons licence, unless indicated otherwise in a credit line to the material. If material is not included in the article's Creative Commons licence and your intended use is not permitted by statutory regulation or exceeds the permitted use, you will need to obtain permission directly from the copyright holder. To view a copy of this licence, visit http://creativecommons.org/licenses/by/4.0/.

\section{References}

1. Lely JC, Smid GE, Jongedijk RA, Knipscheer W, Kleber RJ. The effectiveness of narrative exposure therapy: a review, metaanalysis and meta-regression analysis. Eur J Psychotraumatol. 2019;10:1550344.

2. Williams ME, Thompson SC. The use of community-based interventions in reducing morbidity from the psychological impact of conflict-related trauma among refugee populations: a systematic review of the literature. J Immigr Minor Health. 2011;13:780-94.

3. Robjant K, Fazel M. The emerging evidence for narrative exposure therapy: a review. Clin Psychol Rev. 2010;30:1030-9.

4. Eurostat E: gas retail tables H1. European Comission; 2016

5. Tribe RH, Sendt KV, Tracy DK. A systematic review of psychosocial interventions for adult refugees and asylum seekers. J Mental Health. 2019;28:662-76.

6. UNHCR W UNAIDS. Policy statement on HIV testing and counselling in health facilities for refugees, internally displaced persons and other persons of concern to UNHCR. Geneva; 2014.

7. Unhcr R. Syria regional refugee response. Turkey: UNHCR Interagency Information Sharing Portal; 2016.

8. Gwozdziewycz N, Mehl-Madrona L. Meta-analysis of the use of narrative exposure therapy for the effects of trauma among refugee populations. Perm J. 2013;17:70.

9. Mahoney A, Karatzias T, Hutton P. A systematic review and metaanalysis of group treatments for adults with symptoms associated with complex post-traumatic stress disorder. J Affect Disord. 2019;243:305-21.

10. Pompili M, Gibiino S, Innamorati M, Serafini G, Del Casale A, De Risio L, Sher L. Prolactin and thyroid hormone levels are associated with suicide attempts in psychiatric patients. Psychiatry Res. 2012;200(2-3):389-94.

11. Pompili M, Shrivastava A, Serafini G, Innamorati M, Milelli M, Erbuto D, Lester D. Bereavement after the suicide of a significant other. Indian J Psychiatry. 2013;55(3):256.

12. Slobodin O, de Jong JT. Family interventions in traumatized immigrants and refugees: a systematic review. Transcult Psychiatry. 2015;52:723-42.

13. Acarturk C, Konuk E, Cetinkaya M, Senay I, Sijbrandij M, Cuijpers P, Aker T. EMDR for Syrian refugees with posttraumatic stress disorder symptoms: results of a pilot randomized controlled trial. Eur J Psychotraumatol. 2015;6:27414.

14. Acarturk C, Konuk E, Cetinkaya M, Senay I, Sijbrandij M, Gulen B, Cuijpers P. The efficacy of eye movement desensitization and reprocessing for post-traumatic stress disorder and depression 
among Syrian refugees: results of a randomized controlled trial. Psychol Med. 2016;46:2583-93.

15. Adenauer H, Catani C, Gola H, Keil J, Ruf M, Schauer M, Neuner F. Narrative exposure therapy for PTSD increases top-down processing of aversive stimuli-evidence from a randomized controlled treatment trial. BMC Neurosci. 2011;12:127.

16. Buhmann CB, Nordentoft M, Ekstroem M, Carlsson J, Mortensen EL. The effect of flexible cognitive-behavioural therapy and medical treatment, including antidepressants on post-traumatic stress disorder and depression in traumatized refugees: pragmatic randomized controlled clinical trial. Br J Psychiatry. 2016;208:252-9.

17. Carlsson J, Sonne C, Vindbjerg E, Mortensen EL. Stress management versus cognitive restructuring in trauma-affected refugees: a pragmatic randomized study. Psychiatry Rec. 2018;266:116-23.

18. Hensel-Dittmann D, Schauer M, Ruf M, Catani C, Odenwald M, Elbert T, Neuner F. Treatment of traumatized victims of war and torture: a randomized controlled comparison of narrative exposure therapy and stress inoculation training. Psychother Psychosom. 2011;80:345-52.

19. Hijazi AM, Lumley MA, Ziadni MS, Haddad L, Rapport LJ, Arnetz BB. Brief narrative exposure therapy for posttraumatic stress in Iraqi refugees: a preliminary randomized clinical trial. J Trauma Stress. 2014;27:314-22.

20. Hinton DE, Pham T, Tran M, Safren SA, Otto MW, Pollack MH. CBT for Vietnamese refugees with treatment-resistant PTSD and panic attacks: a pilot study. J Trauma Stress. 2004;17:429-33.

21. Hinton DE, Chhean D, Pich V, Safren SA, Hofmann SG, Pollack $\mathrm{MH}$. A randomized controlled trial of cognitive-behavior therapy for Cambodian refugees with treatment-resistant PTSD and panic attacks: a cross-over design. J Trauma Stress. 2005;18:617-29.

22. Neuner F, Kurreck S, Ruf M, Odenwald M, Elbert T, Schauer M. Can asylum-seekers with posttraumatic stress disorder be successfully treated? A randomized controlled pilot study. Cogn Behav Therapy. 2010;39:81-91.

23. Paunovic N, Öst LG. Cognitive-behavior therapy vs exposure therapy in the treatment of PTSD in refugees. Behav Res Ther. 2001;39:1183-97.

24. Sonne C, Carlsson J, Bech P, Vindbjerg E, Mortensen EL, Elklit A. Psychosocial predictors of treatment outcome for traumaaffected refugees. Eur J Psychotraumatol. 2016;7:30907.

25. Stenmark H, Catani C, Neuner F, Elbert T, Holen A. Treating PTSD in refugees and asylum seekers within the general health care system. A randomized controlled multicenter study. Behav Res Ther. 2013;51:641-7.

26. ter Heide FJJ, Mooren T, Kleijn W, de Jongh A, Kleber R. EMDR versus stabilisation in traumatised asylum seekers and refugees: results of a pilot study. Eur J Psychotraumatol. 2011;2:5881.

27. Wang SJ, Bytyçi A, Izeti S, Kallaba M, Rushiti F, Montgomery E, Modvig J. A novel bio-psycho-social approach for rehabilitation of traumatized victims of torture and war in the post-conflict context: a pilot randomized controlled trial in Kosovo. Conflict Health. 2017;10:34.

28. Drožđek B, Bolwerk N. Evaluation of group therapy with traumatized asylum seekers and refugees: the Den Bosch Model. Traumatology. 2010;16:117-27.

29. Droždek B, Kamperman AM, Bolwerk N, Tol WA, Kleber RJ. Group therapy with male asylum seekers and refugees with posttraumatic stress disorder: a controlled comparison cohort study of three day-treatment programs. J Nerv Ment Dis. 2012;200(9):758-65.

30. Lakshimi Vijayakumar L. Challenges and opportunities in suicide prevention in South-East Asia. WHO South-East Asia J Public Health. 2017;6:45-9.

31. Weinstein N, Khabbaz F, Legate N. Enhancing need satisfaction to reduce psychological distress in Syrian refugees. J Consult Clin Psychol. 2016;84:645.

32. Brune M, José Eiroá-Orosa F, Fischer-Ortman J, Haasen C. Effectiveness of psychotherapy for traumatized refugees without a secure residency status. Intl J Migrat Health Social Care. 2014;10:52-9.

33. Carlsson JM, Olsen DR, Kastrup M, Mortensen EL. Late mental health changes in tortured refugees in multidisciplinary treatment. J Nerv Ment Dis. 2010;198:824-8.

34. d'Ardenne P, Ruaro L, Cestari L, Fakhoury W, Priebe S. Does interpreter-mediated CBT with traumatized refugee people work? A comparison of patient outcomes in East London. Behav Cogn Psychother. 2007;35:293-301.

35. Drožđek B, Kamperman AM, Tol WA, Knipscheer JW, Kleber RJ. Seven-year follow-up study of symptoms in asylum seekers and refugees with PTSD treated with trauma-focused groups. J Clin Psychol. 2014;70:376-87.

36. Halvorsen JØ, Stenmark H. Narrative exposure therapy for posttraumatic stress disorder in tortured refugees: a preliminary uncontrolled trial. Scand J Psychol. 2010;51:495-502.

37. Raghavan S, Rasmussen A, Rosenfeld B, Keller AS. Correlates of symptom reduction in treatment-seeking survivors of torture. Psychol Trauma. 2013;5:377.

38. Sierre van Wyk S, Schweitzer R, Brough M, Vromans L, Murray $\mathrm{K}$. A longitudinal study of mental health in refugees from Burma: the impact of therapeutic interventions. Aust N Z J Psychiatry. 2012;46:995-1003.

39. Whitsett D, Sherman MF. Do resettlement variables predict psychiatric treatment outcomes in a sample of asylum-seeking survivors of torture? Int J Soc Psychiatry. 2017;63:674-85.

40. Heeren M, Wittmann L, Ehlert U, Schnyder U, Maier T, Müller J. Psychopathology and resident status: comparing asylum seekers, refugees, illegal migrants, labor migrants, and residents. Compr Psychiatry. 2014;55(4):818-25.

41. Fondazione I. S. M. U. Ventitreesimo Rapporto sulle migrazioni 2017; Franco Angeli; 2017.

42. Norton PJ. Depression Anxiety and Stress Scales (DASS-21): psychometric analysis across four racial groups. Anxiety Stress Coping. 2007;20:253-65.

43. Rohlof HG, Knipscheer JW, Kleber RJ. Somatization in refugees: a review. Soc Psychiatry Psychiatr Epidemiol. 2014;49:1793-804.

44. Pennebaker JW, Evans JF. Expressive writing: words that heal: using expressive writing to overcome traumas and emotional upheavals, resolve issues, improve health, and build resilience. Enumclaw: Idyll Arbor; 2014.

Publisher's Note Springer Nature remains neutral with regard to jurisdictional claims in published maps and institutional affiliations. 